DFF 288/10/97

hep-th/9710248

\title{
Isomonodromic Properties of the Seiberg-Witten Solution
}

\author{
Andrea CAPPELLI, Paolo VALTANCOLI and Luca VERGNANO \\ I.N.F.N. and Dipartimento di Fisica \\ Largo E. Fermi 2, I-50125 Firenze, Italy
}

\begin{abstract}
The Seiberg-Witten solution of $N=2$ supersymmetric $S U(2)$ gauge theories with matter is analysed as an isomonodromy problem. We show that the holomorphic section describing the effective action can be deformed by moving its singularities on the moduli space while keeping their monodromies invariant. Well-known examples of isomonodromic sections are given by the correlators of two-dimensional rational conformal field theories - the conformal blocks. The Seiberg-Witten section similarly admits the operations of braiding and fusing of its singularities, which obey the Yang-Baxter and Pentagonal identities, respectively. Using them, we easily find the complete expressions of the monodromies with affine term, and the full quantum numbers of the BPS spectrum. While the braiding describes the quark-monopole transmutation, the fusing implies the superconformal points in the moduli space. In the simplest case of three singularities, the supersymmetric sections are directly related to the conformal blocks of the logarithmic minimal models.
\end{abstract}

October 1997 


\section{Introduction}

The beautiful exact solution of the low-energy effective actions of $N=2$ supersymmetric gauge theories in four dimensions [1] has led to dramatic developments in field theory and string theory over the last three years [2]. In this paper, we would like to analyse some mathematical aspects of the exact solution and compare them with analogous properties of the correlators of two-dimensional conformal field theory a well-understood exactly solvable problem [3]. Our general motivation is to understand the integrable structure behind the Seiberg-Witten solution and to develop a framework for extending the solution to other field-theory observables. We shall make a few steps in this program, which we find interesting, and, meanwhile, we shall obtain some physical results for the spectrum of these theories. We shall consider the simple case of $N=2$ supersymmetric $S U(2)$ gauge theories with $N_{f}$ massive quark hypermultiplets, $N_{f}=0,1,2,3$ [1].

A crucial element of the $N=2$ supersymmetric solution is the holomorphicity of the prepotential $\mathcal{F}(\Psi)$ as a functional of $N=2$ chiral superfield $\Psi$ 世. This is a consequence of chiral decomposition of supersymmetric representations: in formulaeף,

$$
\bar{D}_{\alpha}^{i} \mathcal{F}(\Psi)=0 \quad \text { and } \quad \bar{D}_{\alpha}^{i} \Psi=0 \quad \longrightarrow \quad \frac{\delta}{\delta \bar{\Psi}} \mathcal{F}(\Psi)=0
$$

Once the fields have acquired a v.e.v., $\langle\Psi\rangle=a$, holomorphicity in field space implies that $\mathcal{F}(a)$ is a holomorphic function of the v.e.v., $\partial / \partial \bar{a} \mathcal{F}(a)=0$, and of the coordinates of the moduli space as well.

A similar property of holomorphicity is found in two-dimensional conformal field theories, if one compares the moduli space of the four-dimensional theories with the coordinate space $z=x_{1}+i x_{2}$ of the two-dimensional theories. Actually, conformal symmetry implies that the stress-energy tensor is holomorphic $\partial / \partial \bar{z} T(z)=0$. This relation is stable under analytic reparametrizations of the coordinate $(z \rightarrow w)$,

$$
\frac{\partial}{\partial \bar{z}} T(z)=0 \leftrightarrow \frac{\partial}{\partial \bar{w}} T(w)=0, \quad z=\sum_{n \in \mathbf{Z}} \epsilon_{n} w^{n+1}
$$

and leads to powerful Ward identities which can be solved for the correlators [3]. Within this infinite-dimensional covariance of the theory, the true symmetry transformations are given by the projective $\operatorname{sl}(2, \mathbf{C})$ subalgebra. To summarize, in conformal

\footnotetext{
${ }^{*}$ In the $N=1$ action, the superpotential $\mathcal{W}(\phi)$ and the gauge effective coupling $\tau(\phi)$ are similarly holomorphic in the $N=1$ chiral superfield $\phi$.

$\dagger$ In Eq.(1.1), $\alpha=1,2$ and $i=1,2$ are the spinor and supersymmetry indices, respectively.
} 
field theory holomorphicity is associated with an infinite-dimensional covariance and integrability.

In the supersymmetric theories, holomorphicity is similarly stable under analytic field redefinitions $(\Psi \rightarrow \Sigma)$ :

$$
\frac{\delta}{\delta \bar{\Psi}} \mathcal{F}(\Psi)=0 \quad \leftrightarrow \quad \frac{\delta}{\delta \bar{\Sigma}} \mathcal{F}(\Sigma)=0, \quad \Psi=\sum_{n \in \mathbf{Z}} \epsilon_{n} \Sigma^{n+1}
$$

These non-linear transformations are not familiar in field theory, because we usually consider the Ward identities for linear field variationsf. The true symmetries of the action are restricted to the finite-dimensional supersymmetric transformations $\delta_{\xi} \Psi=$ $\xi^{i \alpha} Q_{\alpha}^{i} \Psi$ which act trivially on the moduli, $\delta_{\xi} a=0$.

Nevertheless, we believe that there should exist Ward identities for the infinitedimensional covariance in field space. A necessary condition for this covariance is the possibility of continuously deforming the moduli space of the low-energy theory, without changing its main features. This is called the isomonodromy property [5]. In this paper, we show that the Seiberg-Witten solution is indeed isomonodromic and discuss some consequent effects.

The Seiberg-Witten solutions of the massive $S U(2)$ theories involve the holomorphic sections $\left(a_{D}(u), a(u)\right)$, whose $\left(N_{f}+2\right)$ singularities can be displaced in the moduli space $\{u \in \mathbf{C}\}$, without varying the monodromies around thems. Actually, the $N_{f}$ quark singularities $u \simeq O\left(m_{i}^{2}\right)$ can be moved by varying the masses, and their monodromies are given by constant integer matrices. The two additional singularities $u= \pm O\left(\Lambda^{2}\right)$ also have integer monodromies, but there is only one parameter for displacing them. Therefore, we should allow a further isomonodromic deformation of the $u$ variable, which changes the physical moduli space into a more general, unphysical one. This can be done because the Seiberg-Witten section is specified by an elliptic curve: small $u$ deformations correspond to small variations of the coefficients of the curve which do not generically change the monodromies. In the particular case of the pure $S U(2)$ theory, it is also possible to displace the two singularities by $S L(2, \mathbf{C})$ projective transformations of $u$, which are invertible in the whole plane and thus leave the monodromies invariant. We thus conclude that the $S U(2)$ Seiberg-Witten solution is "covariant" under $S L(2, \mathbf{C})$ transformations in the $u$ plane.

The isomonodromy of the Seiberg-Witten solution has also been discussed in the

$\ddagger$ In particular, non-linear transformations of $N=2$ chiral fields are usually disregarded [4], because they violate the Bianchi identity for the photon in the $N=2$ chiral multiplet $\left(D^{i \alpha} D_{\alpha}^{j} \Psi=\right.$ $\left.\bar{D}_{\dot{\alpha}}^{i} \bar{D}^{j \dot{\alpha}} \bar{\Psi}\right)$.

$\S$ Another singularity sits at infinity. 
recent Refs. [6] [7]. This is a general property of integrable systems, which is not too useful in practice: one actually needs to identify the specific class of integrable systems corresponding to the Seiberg-Witten solution, and to characterize it by an infinite-dimensional covariance. Some interesting relations with integrable systems have been found in the Refs. 8 .

The rational conformal field theories are a well-understood class of isomonodromy problems. The monodromy properties of the $n$-point conformal blocks (holomorphic part of the correlators) have been analysed by Moore and Seiberg in Ref. [9]. Consider for example the holomorphic part of the four-point function:

$$
\left\langle\phi_{1}\left(z_{1}\right) \phi_{2}\left(z_{2}\right) \phi_{3}\left(z_{3}\right) \phi_{4}\left(z_{4}\right)\right\rangle \propto \sum_{j=1}^{q} a_{j} \mathcal{F}_{i_{1} i_{2} i_{3} i_{4}}^{j}\left(\frac{\left(z_{1}-z_{2}\right)\left(z_{3}-z_{4}\right)}{\left(z_{1}-z_{3}\right)\left(z_{2}-z_{4}\right)}\right) .
$$

In this equation, the $q$ conformal blocks $\mathcal{F}^{j}(\eta)$ form a $q$-dimensional holomorphic section with three branch points at $\eta=0,1, \infty$, which satisfy a $q$-th order differential equation. The behaviour of each block $\mathcal{F}^{j}$ for $z_{1} \rightarrow z_{2}$ is given by the corresponding term in the operator product expansion,

$$
\phi_{1}\left(z_{1}\right) \phi_{2}\left(z_{2}\right) \simeq \sum_{j=1}^{q}\left(z_{1}-z_{2}\right)^{h_{j}-h_{1}-h_{2}} \phi_{j}\left(z_{2}\right),
$$

where $h_{1}, h_{2}, h_{j}$ are the conformal dimensions of the fields. This fusion of fields is described by the "dual" diagram in Fig.(11). The monodromy for $z_{1}$ going around $z_{2}$ has the diagonal form $M_{2}=\left(\Lambda_{2}\right)_{j j^{\prime}}=\delta_{j j^{\prime}} e^{i 2 \pi\left(h_{j}-h_{1}-h_{2}\right)}$; it is independent of the position of the other singularities, because they do not enter in the local operatorproduct expansion. The monodromies around the other singularities are of the form $M_{i}=U_{i} \Lambda_{i} U_{i}^{-1}$, where the $U_{i}$ are the transformations for carrying the monodromy paths to a common base point. The diagonal matrices $\Lambda_{i}$ are again determined by the operator-product expansion of the corresponding fields; the $U_{i}$ are the transformations among equivalent sets of solutions of the differential equation for $\mathcal{F}^{j}$, which are determined by the universal data of the rational conformal field theory [10]. Therefore, all the monodromies are independent of the $\left\{z_{i}\right\}$.

Moore and Seiberg have introduced the fusion operator which acts on the fields and modifies the fusion pattern; namely, it replaces the "s-channel" diagram in Fig.(1) with the "t-channel" diagram. Furthermore, there is the braiding operator which exchanges the fields in the block by analytic continuation in the $z$-plane. This operator satisfies the Yang-Baxter identity due to the associativity of exchanges; moreover, the associativity of the fusion and braiding operators is enforced by the MooreSeiberg Pentagonal identity. The Yang-Baxter and Pentagonal identities summarize the isomonodromy of the conformal blocks. 


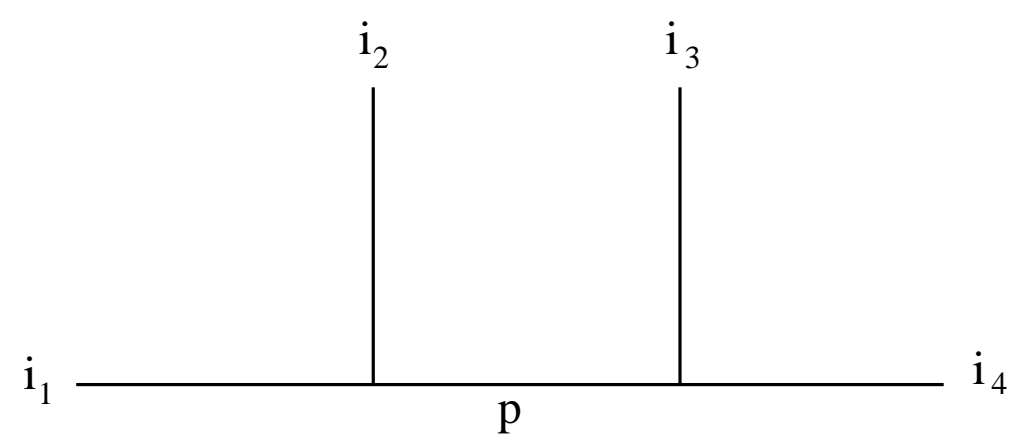

Figure 1: "Duality" diagram for the 4-point conformal block.

In this paper, we show that analogous braiding and fusing operations can be defined for the Seiberg-Witten sections. On the other hand, we also find that these holomorphic functions are different from the conformal sections, in general. In Section 2 , we follow the motion of the singularities as the quark masses are varied and find that they braid and fuse in pairs. We define the braiding and fusing operators which act on the singularities, rather than the "fields", and actually transform their monodromy matrices. We then show that these operators satisfy the Yang-Baxter and Pentagonal identities, respectively. Actually, these identities express topological properties which must hold in any isomonodromy problem.

The physical meaning of these braidings and fusings in the supersymmetric theory is the following [1]: the braiding describes the transmutation of a massless quark singularity at weak coupling $\left(u \simeq m^{2}\right.$ for $\left.m^{2} \gg \Lambda^{2}\right)$ into a massless monopole one at strong coupling $\left(u=O\left(\Lambda^{2}\right)\right.$ for $\left.m^{2}=O\left(\Lambda^{2}\right)\right)$. The fusing describes the merging of quark and monopole singularities into a superconformal singular point [1]. These critical points occur sometimes at finite values $m_{i}=O(\Lambda)$ on the trajectory $m_{i} \rightarrow \infty$ of a quark decoupling - a rather odd renormalization group behaviour. Actually, we show that the pattern of superconformal points, i.e. of the fusings, is determined by the consistency with the braidings; namely, it is a physical effect of isomonodromy.

The BPS mass formula for $N=2$ supersymmetric theories with $N_{f}$ quark hypermultiplets of mass $m_{f}$ is given by [1]:

$$
m^{2}=2|Z|^{2}, \quad Z=n_{m} a_{D}(u)+n_{e} a(u)+\sum_{f=1}^{N_{f}} s_{f} \frac{m_{f}}{\sqrt{2}},
$$

where $\left(n_{m}, n_{e}, s_{f}\right)$ are the magnetic, electric and (pseudo)-baryonic quantum numbers, respectively. The monodromy transformations of $\left(a_{D}, a\right)$ contain additive terms proportional to the masses, which were not completely understood in the literature. In Section 2.3, we use the Yang-Baxter and Pentagonal identities as equations to de- 
termine completely these affine terms; as an input, we use the matching between the $N_{f}$ and the $\left(N_{f}-1\right)$ theories at the quark decouplings. This algebraic derivation is simpler than the direct analytic continuation of the general Seiberg-Witten sections. The complete monodromy matrices of the $N_{f}=1$ theory are derived in the text, while those of the $N_{f}=2$ and 3 theories are found in the Appendix A. Moreover, simple explicit expressions for the Seiberg-Witten sections are obtained in Appendix $\mathrm{B}$ for the cases of maximally fused singularities; their monodromy transformations are then used to check the results by the algebraic approach.

Next, we use the complete monodromies to determine the full spectrum of the pseudo-baryonic quantum numbers. The $\left(n_{m}, n_{e}, s_{f}\right)$ quantum numbers in (1.6) are also transformed under the monodromy of $\left(a_{D}, a\right)$, so that $Z$ remains invariant; this is the spectral flow, which should map the spectrum into itself. Following Ref. [12], we use this flow as a self-consistent constraint which determines the complete weakcoupling spectrum.

In Section 3, we study more closely the analytic properties of the Seiberg-Witten section in the first non-trivial case of three singularities $\left(N_{f}=1\right)$. We rewrite the Picard-Fuchs equations satisfied by the section [13] into a Fuchsian second-order differential equation, which is the traditional setting for studying isomonodromy [14. This property implies a set of differential integrability conditions for the residues of the single-pole terms (the accessory parameters) and the apparent singularity in the Fuchsian equation. For three singularities, these conditions reduces to a single nonlinear differential equation, the Painlevé VI equation for the apparent singularity. We find that this is rather remarkably satisfied by the Seiberg-Witten solution: this yields an explicit proof of isomonodromy, which confirms the previous, intuitive arguments. As an outcome of this analysis, we find a new first-order differential equation for the mass variation $\left(\partial a_{D} / \partial m, \partial a / \partial m\right)$.

In Section 4, we directly identify some Seiberg-Witten sections with the conformal blocks of suitable conformal field theories. The two-dimensional monodromies imply the operator product expansion $\phi_{1} \cdot \phi_{2}=\phi+\phi^{\prime}$. This is reproduced, for example, by the primary field $\phi_{1,2}$ of the minimal conformal models with central charge $c\left(p, p^{\prime}\right)=1-$ $6\left(p-p^{\prime}\right)^{2} / p p^{\prime}$. The presence of logarithmic singularities requires that the dimensions of the fields $\phi$ and $\phi^{\prime}$ are equal, $h=h^{\prime}$; this occurs in the so-called logarithmic conformal field theories with $c=c\left(1, p^{\prime}\right)$ 15. We consider the simplest SeibergWitten sections with three singularities, which describe the $N_{f}=0$ theory and $N_{f}=$ 1,2,3 ones with $\left(N_{f}+1\right)$ singularities fused into a superconformal point. Indeed, we are able to identify these sections with the four-point conformal blocks of the theories 
with $c=c(1,2), c(1,3), c(1,4)$ and $c(1,6)$, respectively.

This correspondence is not sufficient to establish the complete equivalence of the supersymmetric and conformal isomonodromy problems. Actually, the previous threesingularity sections are necessarily Hypergeometric functions, which are conformally covariant under the $S L(2, \mathbf{C})$ projective transformations of the $u$-plane, and thus can be represented by (quasi)-primary conformal fields [3]. On the contrary, the general supersymmetric sections with $\left(3+N_{f}\right)$ singularities are shown to be non-covariant under $S L(2, \mathbf{C})$, and thus cannot correspond to conformal blocks. It remains an open question whether the supersymmetric isomonodromy problem can be characterized by an infinite-dimensional covariance. In Appendix C, we recall some general relations among isomonodromy, integrability and conformal symmetry, which complement the analysis of Section 4.

In conclusion, we believe that it is interesting to investigate the relations of the Seiberg-Witten theory with other isomonodromy problems which are not conformal in general, like the topological field theories [16], the matrix models and quantum Liouville theory [17]. Actually, a relation with the topological theories has already been suggested in the Refs. 18] [19]: starting from the supersymmetric effective action, these Authors found an analogue of the fusion rules of the topological fields, which satisfy an associativity condition similar to the one discussed here and encode common isomonodromic properties.

\section{$2 \quad$ Braiding and Fusing of Monodromies and Their Identities}

\subsection{Representation of the Monodromy Group}

We begin by introducing our notation for describing the monodromies. Let us first consider the $N_{f}=0, S U(2)$ theory for simplicity, whose section $\left(a_{D}(u), a(u)\right)$ has two singularities located at $u_{1}=\Lambda_{0}$ and $u_{2}=-\Lambda_{0}$. We can represent the section by a two-dimensional vector $\mathbf{a}=\left(a_{D}, a\right)$ and the monodromy transformation by matrix multiplication:

$$
\left(\begin{array}{c}
a_{D} \\
a
\end{array}\right)\left(e^{2 \pi i}\left(u-u_{i}\right)+u_{i}\right)=M_{i}\left(\begin{array}{c}
a_{D} \\
a
\end{array}\right), \quad i=1,2 .
$$

The corresponding transformation of the vector of magnetic and electric charges $\mathbf{n}=$ $\left(n_{m}, n_{e}\right)$ is by right multiplication $\mathbf{n} \rightarrow \mathbf{n} M_{i}^{-1}$, such that the BPS mass formula 


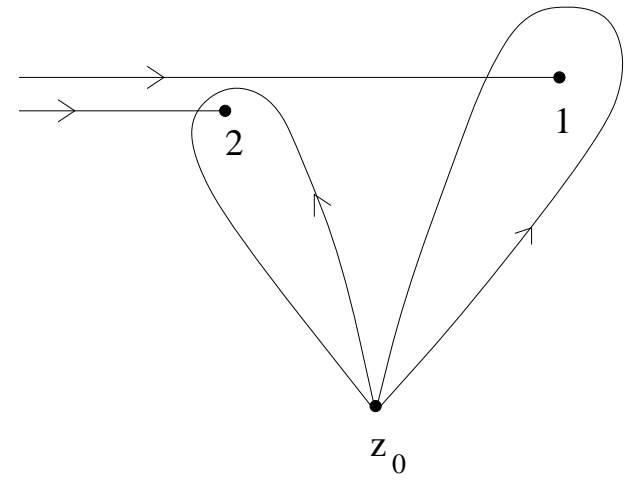

(a)

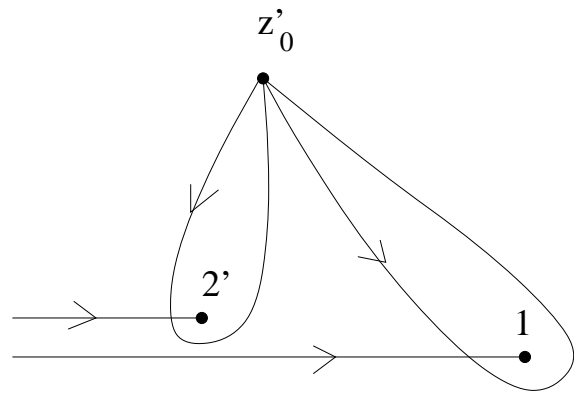

(b)

Figure 2: Description of the monodromies for the $N_{f}=0$ theory.

$Z=\mathbf{n} \cdot \mathbf{a}$ in Eq.(1.6) remains invariant. We recall from Ref.[12] the monodromy matrices $\nabla^{\pi}$ :

$$
M_{1}=\left(\begin{array}{cc}
1 & 0 \\
-1 & 1
\end{array}\right), M_{2}=\left(\begin{array}{cc}
3 & 4 \\
-1 & -1
\end{array}\right), M_{2^{\prime}}=\left(\begin{array}{ll}
-1 & 4 \\
-1 & 3
\end{array}\right),
$$

which satisfy the relations,

$$
M_{1} M_{2^{\prime}}=M_{2} M_{1}=M_{\infty}, \quad M_{\infty}=\left(\begin{array}{cc}
-1 & 4 \\
0 & -1
\end{array}\right) \equiv M_{\infty}^{(0)} .
$$

These non-Abelian monodromies depend on the position of the base point for the loop and on the location of the cuts. As explained in Ref. [12], the analytic continuation of the section $\left(a_{D}, a\right)$ depends on the Riemann sheet of $u \sim u_{i}$ in Eq.(2.1): for example, there are two possibilities $M_{2}$ and $M_{2^{\prime}}$ for the second singularity, due to presence of the cut emanating from the first singularity. In principle, one should keep track of the Riemann sheets when composing the monodromies.

Our representation of the monodromies is shown in Fig.(2): the two elementary loops of Fig.(2a) can be associated to the monodromies $M_{1} \equiv M_{1}\left(z_{0}\right)$ and $M_{2} \equiv M_{2}\left(z_{0}\right)$, because they reproduce $M_{\infty}=M_{2} M_{1}$ by composition; similarly, the monodromies $M_{1}$ and $M_{2^{\prime}} \equiv M_{2}\left(z_{0}^{\prime}\right)$ correspond to the loops in Fig.(26) $\mathrm{b}$. Our graphical rules do not faithfully reproduce all the analytic properties of the Seiberg-Witten

\footnotetext{
『 In the normalization valid for $N_{f}>0$ [1].
} 


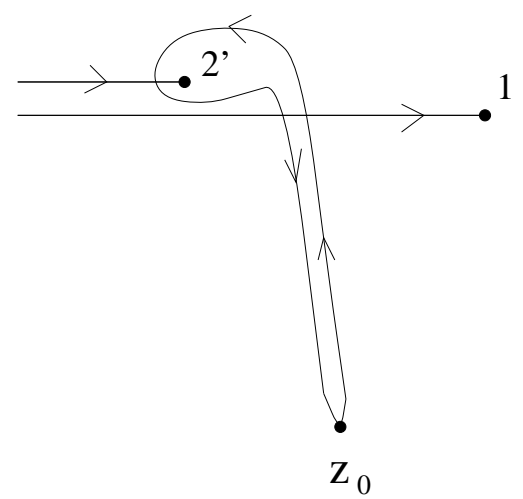

(a)

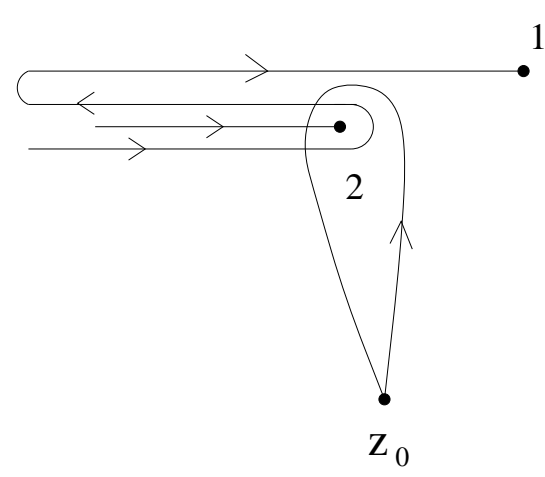

(b)

Figure 3: Changes of patch

section, but will be sufficient to describe the monodromy group and the braiding and fusing operators; they are inspired by the previous analysis of Chern-Simons monodromies in a "singular gauge" 20]:

- We use a single plane with cuts, which corresponds to an initial, conventional choice of the Riemann sheet, i.e. of the patch and its monodromies;

- We can imagine that all the non-trivial monodromy is concentrated at the crossings of the path with the cuts: this could be obtained by an analytic reparametrization of the $u$ coordinate, which leaves the monodromies invariant [20];

- The monodromies are invariant if the base point is moved in the plane without crossing a cut;

- The monodromies with fixed base point are invariant under the motion of the singularities: the deformed paths following them can cross a cut but not a singularity.

In Fig. 苟, we use these rules to deal with: $(a)$, the change of base point; $(b)$, the motion of the singularity (2) through the cut of (1). In order to compare $M_{2}$ with $M_{2^{\prime}}$, we imagine to move (2) in Fig.(22a) above the cut of (1): since $M_{2}\left(z_{0}\right)$ is invariant, we find that

$$
M_{2}\left(z_{0}\right)=M_{1} M_{2^{\prime}} M_{1}^{-1} \longrightarrow M_{2^{\prime}}=M_{1}^{-1} M_{2} M_{1},
$$

which is in agreement with (2.3). Fig.(阿) describes the opposite motion of $\left(2^{\prime}\right)$ going above the cut of (1), and shows that the singularity (2) can be obtained by "dressing" 
$\left(2^{\prime}\right)$, which amounts to the conjugation (2.4) again. Finally, we choose to send all the cuts to infinity along the same direction; in this case, $M_{\infty}$ is independent of the base point.

\subsection{Braiding and Fusing Operators and "Duality" Identities}

Let us first consider the $N_{f}=1, S U(2)$ theory as an example; the properties of the Seiberg-Witten solution have been analysed in Refs. 11] 12, which contains some useful background. The elliptic curve is:

$$
y^{2}=x^{3}-u x^{2}+m \frac{\Lambda_{1}^{3}}{4} x-\frac{\Lambda_{1}^{6}}{64} .
$$

This determines the Seiberg-Witten section $\left(a_{D}(u), a(u)\right)$ by $u$-integration of the Abelian integrals,

$$
\frac{d a}{d u}=\frac{\sqrt{2}}{8 \pi} \oint_{\gamma_{1}} \frac{d x}{y}, \quad \frac{d a_{D}}{d u}=\frac{\sqrt{2}}{8 \pi} \oint_{\gamma_{2}} \frac{d x}{y},
$$

and by matching with the known asymptotics. The singularities in the $u$-plane occur when one period $\gamma_{i}$ vanishes and are given by the zeroes of the discriminant of the curvell (2.5):

$$
\Delta(u)=8\left(u^{3}-u^{2} m^{2}-9 u m+8 m^{3}+\frac{27}{4}\right) .
$$

The singularities for $m=0$ are located at $u=\left(u_{0} e^{i \pi / 3},-u_{0}, u_{0} e^{-i \pi / 3}\right)$, with $u_{0}=$ $3 / 4^{1 / 3}$. When the mass is switched on, their motion in the $u$-plane is shown in Fig.(四): for real positive mass, (case (a)), the singularities (1) and (3) fuse together at $u=u_{c}=3$ and $m=m_{c}=3 / 2$, and then split again and move on the real axis. The $\left(u_{c}, m_{c}\right)$ point describes a superconformal theory, which has been discussed in the Refs. [11. After the split, one singularity approaches the value $u \simeq m^{2}$ for large $m$, which corresponds to the weak-coupling quark singularity. The other two singularities asymptotically become those of the $N_{f}=0$ theory: actually, after matching the $\Lambda$-parameters by $\Lambda_{0}^{4}=m \Lambda_{1}^{3}$, their position tends to $u= \pm \Lambda_{0}^{2}$. This motion of singularities satisfies the expected "renormalization-group flow" "* from the $N_{f}$ theory to the $\left(N_{f}-1\right)$ theory when one quark becomes very massive and decouples. Note, however, the rather unusual presence of a critical point at finite bare mass, whose origin will be explained later.

\footnotetext{
" Hereafter, we shall often use the normalization $\Lambda_{N_{f}}^{4-N_{f}}=8$.

** Actually, this is the explicit tuning of a parameter.
} 

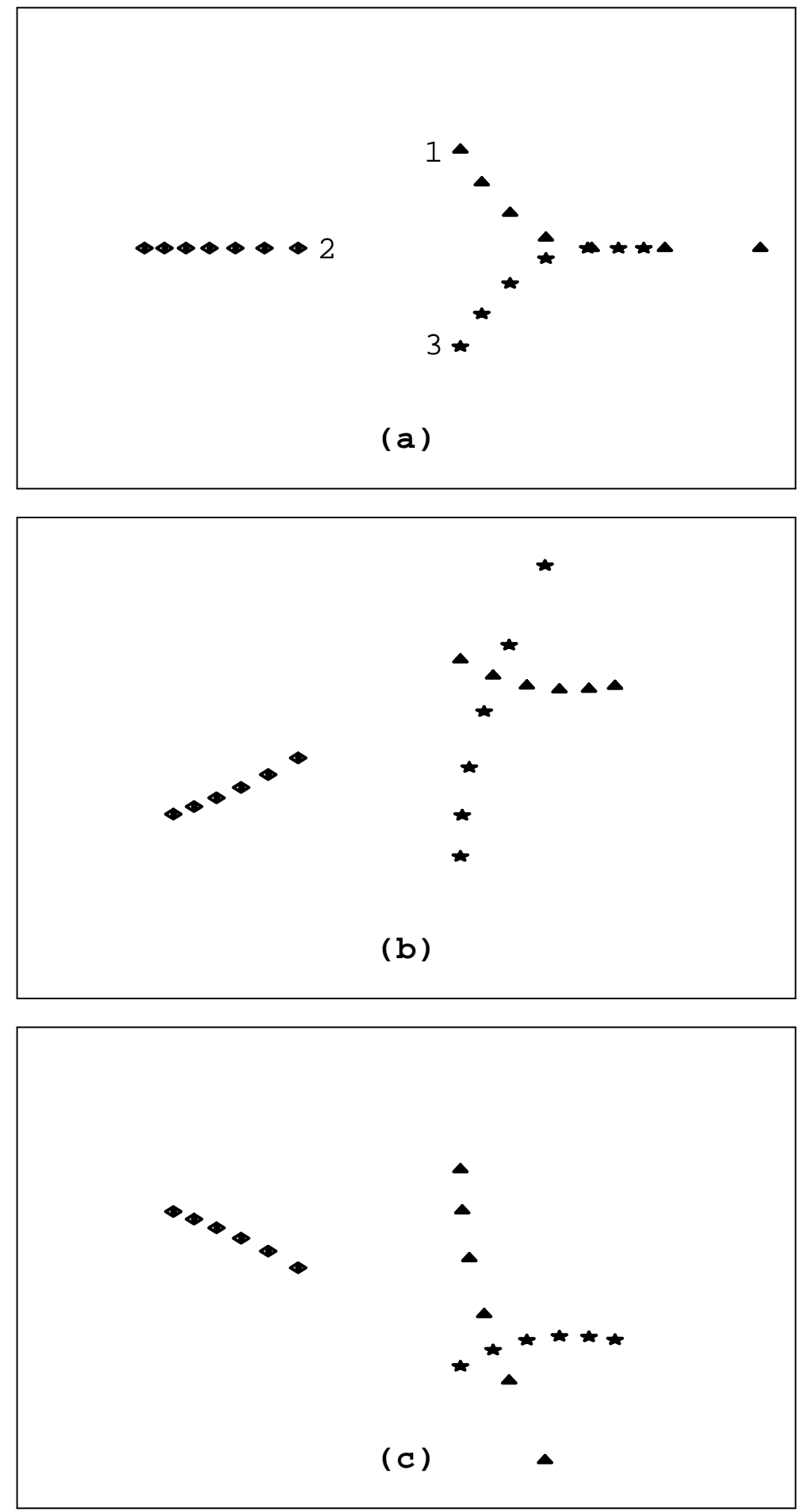

Figure 4: Motion of the three $N_{f}=1$ singularities in the $u$-plane: $(a), \operatorname{Arg}(m)=0$; (b), $\operatorname{Arg}(m)>0$; and $(c), \operatorname{Arg}(m)<0$. 
Fig.(4) $\mathrm{b}$ ) shows the motion of the singularities for $m$ growing along the ray $\operatorname{Arg}(m)=$ $\pi / 6$ ( Fig. 1 (c) is the case $\operatorname{Arg}(m)=-\pi / 6$ ). Here one sees that the trajectories of the singularities (1) and (3) cross each other before approaching the decoupling asymptotic configurations $u= \pm \sqrt{m \Lambda_{1}^{3}}$ and $u=m^{2}$, on the asymptotic rays $\operatorname{Arg}(u)=\operatorname{Arg}(m) / 2$ and $\operatorname{Arg}(u)=2 \operatorname{Arg}(m)$, respectively. These trajectories corresponds to an exchange of the singularities, which pass by the crossing point at different $m$ values. One can see that the singularities braid clockwise or counterclockwise for $\operatorname{Arg}(m)>0$ and $\operatorname{Arg}(m)<0$, respectively. Note also that the braiding and fusing of other pairs of singularities similarly occurs for $\pi / 3<\operatorname{Arg}(m)<\pi$ and $-\pi<\operatorname{Arg}(m)<-\pi / 3$, due to the $\mathbf{Z}_{3}$ symmetry of the $m=0$ theory.

Similar braidings and fusings of the singularities of an holomorphic section have been analysed in conformal field theory by Moore and Seiberg [9]. They were able to define the operators which specify the analytic continuation and the fusion of the fields in the conformal blocks, respectively. Here, we do not have a representation of the Seiberg-Witten section in terms of "fields" (see however Section 4); therefore, we shall describe the simpler braiding and fusing operators acting on the monodromy matrices themselves.

In the theories with massive quarks, the monodromy transformations acquire an additive term proportional to the mass [1]; for the sake of simplicity, we shall first discuss the monodromies of $\left(d a_{D} / d u, d a / d u\right)$, which do not have this term. Let us also recall from Ref. 12] the general formula for the monodromy around the singularity caused by $k$ BPS states with charge $\mathbf{n}=\left(n_{m}, n_{e}\right)$ becoming massless:

$$
M_{k\left(n_{m}, n_{e}\right)}=\left(\begin{array}{cc}
1+n_{e} n_{m} k & n_{e}^{2} k \\
-n_{m}^{2} k & 1-n_{e} n_{m} k
\end{array}\right) .
$$

These are the "elementary" monodromies, made by the analytic continuation along a small loop near the singularity. The following properties:

$$
M_{k\left(n_{m}, n_{e}\right)}=M_{k\left(-n_{m},-n_{e}\right)}=M_{k / \ell^{2}\left(\ell n_{m}, \ell n_{e}\right)}, \quad M_{k\left(n_{m}, n_{e}\right)}^{-1}=M_{-k\left(n_{m}, n_{e}\right)},
$$

imply a one-to-one correspondence between monodromy matrices and charge vectors $\mathbf{n}$ for $n_{m} \leq 2$, as in the present theories. We also need the general formula for the conjugation of monodromies:

$$
\begin{aligned}
M_{\left(n_{m}, n_{e}\right)} & \rightarrow M_{k\left(n_{m}^{\prime}, n_{e}^{\prime}\right)}^{-1} M_{\left(n_{m}, n_{e}\right)} M_{k\left(n_{m}^{\prime}, n_{e}^{\prime}\right)}, \\
\left(n_{m}, n_{e}\right) & \rightarrow\left(n_{m}, n_{e}\right)+\left(n_{m}^{\prime}, n_{e}^{\prime}\right) k\left[n_{m} n_{e}^{\prime}-n_{e} n_{m}^{\prime}\right] .
\end{aligned}
$$

This shows that the monodromies of "mutually local" particles $\left(\mathbf{n} \times \mathbf{n}^{\prime}=0\right)$ commute among themselves. 
A convenient choice of patch for the $N_{f}=1$ section is shown in Fig.(5a), following our previous conventions. The elementary monodromies around the three singularities can be carried to a common base point located in the bottom right corner of the figure; their values are [1]:

$$
M_{1}=M_{(1,-1)}, \quad M_{2}=M_{(1,1)}, \quad M_{3}=M_{(1,0)} .
$$

The composition giving the monodromy at infinity is,

$$
M_{2} M_{3} M_{1}=M_{\infty}^{(1)}=\left(\begin{array}{cc}
-1 & 3 \\
0 & -1
\end{array}\right),
$$

as it can be easily recognized from the order of the cuts at infinity. Let us recall that the values of the electric charge are patch dependent, due to the phenomenon of "democracy of dyons" $\square$ : any other choice of three consecutive values in Eq.(2.11) would be equivalent by rotating the $\theta$-angle, i.e. $\operatorname{Arg}(u)$ at infinity. It is important to keep this arbitrariness in mind when comparing different results in the literature.

Let us now describe the transformation of the three monodromies (2.11) corresponding to the counter-clockwise exchange of two singularities in (Fig.(4⿻) $(\operatorname{Arg}(m)<0)$. As shown in Fig. (5a), the singularity $(1,-1)$ crosses the cut of $(1,0)$ and goes into another Riemann sheet. This can be taken into account by deforming the cut and dressing the singularity according to Fig.(5b): the result is a new elementary monodromy around this singularity, Fig.(5c), as seen in the original Riemann sheet, and from the same base point. This is obtained by the conjugation:

$$
M_{(1,-1)} \rightarrow M_{(1,0)} M_{(1,-1)} M_{(1,0)}^{-1}=M_{(0,1)}
$$

Therefore, the massless dyon has become a massless quark. Next, Fig.(5d) shows the configuration which should be attained for the decoupling of this massive quark: its cut should go to infinity as well, such that the moduli space at finite $u\left(\Lambda_{0} \sim\right.$ $u \ll m$ ) only contains the singularities and the cuts of the $N_{f}=0$ theory in Eq.(2.2) and Fig.(2)a). This requires a further conjugation of the singularity (2), yielding $\mathbf{n}_{2}=(1,1) \rightarrow(1,2)$. In conclusion, the quark decoupling is characterized by the following relation between the singularities at infinity in the two theories:

$$
M_{\infty}^{(1)}=M_{(0,1)} M_{(1,2)} M_{(1,0)}=M_{\text {quark }} M_{\infty}^{(0)}
$$

The two characterizations (2.13) of the same singularity as a dyon and a quark pertain to different Riemann sheets, and there is no discontinuity in the physics.

†† See Ref.[21] for a complete discussion. 


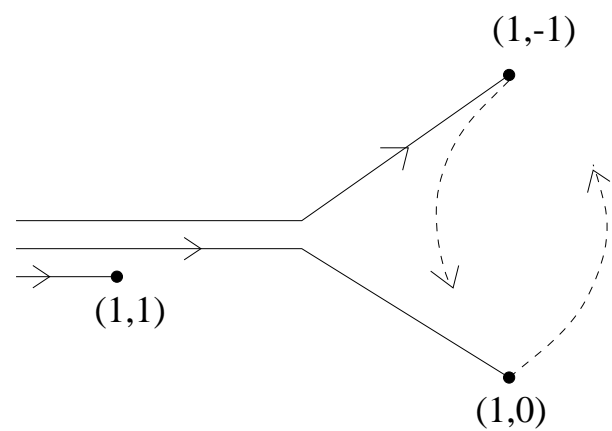

(a)

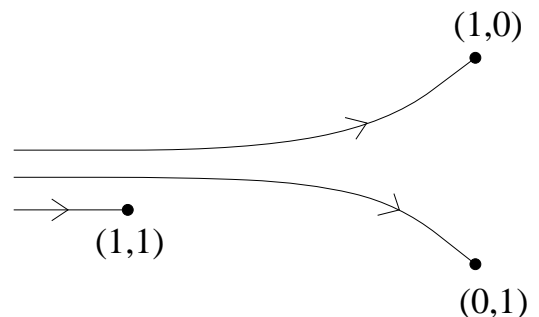

$(0,1)$

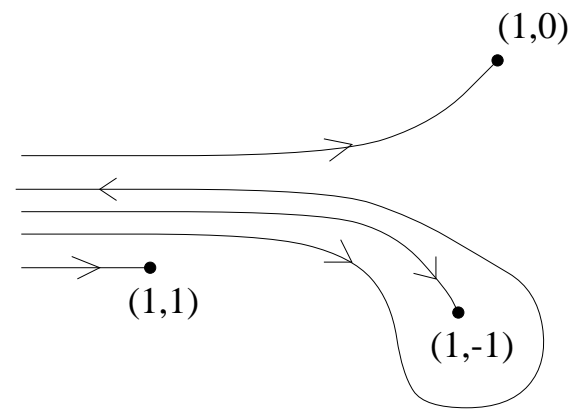

(b)

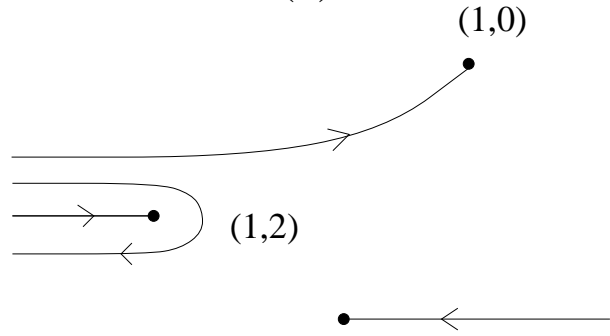

$(0,1)$

(c)

(d)

Figure 5: Deformations of cuts associated to the singularity exchange and the quark decoupling.

Within each patch, it is possible to vary smoothly $u$ from a given point (e.g. the base point), reach any singularity and find that a certain BPS state is massless there. The discontinuity is found when comparing the two asymptotic behaviours, $m \sim \Lambda_{1}$ (in the strong coupling region) and $m \gg \Lambda_{1}$ (in the weak coupling region): these are necessarily described by two different Riemann sheets due to the topology of the motion of the singularities and the cuts. Actually, if we stay always in the same patch, we find that the cuts get tangled and, asymptotically, it becomes impossible to reach smoothly all the singularities from a common point. Therefore, the motion of the singularities implies an asymptotic change in the charges of the massless particles, due to the necessary changes of patches; this is a rather remarkable consequence of the physics being described by a multi-valued holomorphic function. Moreover, upon breaking the $N=2$ supersymmetry to $N=1[\mathbb{1}]$, this property verifies the equivalence of the confinement phase (monopole condensation) and the Higgs phase (quark condensation) in gauge theories with quarks in the fundamental representation [司].

The counter-clockwise exchange of two singularities in Fig.(55a,b) can be associated 


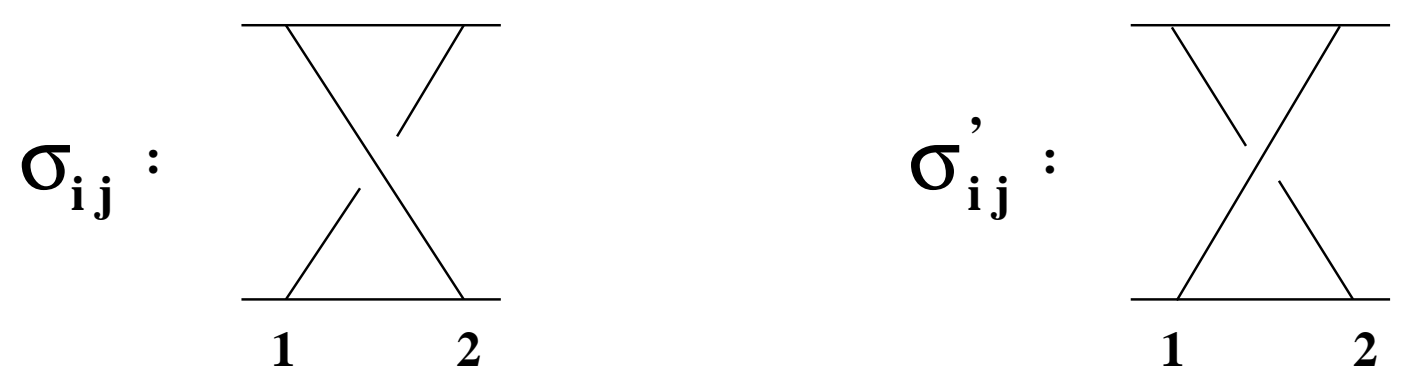

Figure 6: Braid operators $\sigma_{i j}$ and $\sigma_{i j}^{\prime}$.

with one of the braid operators $\sigma_{i j}, i, j=1,2,3$, which act in the tensor product $V_{1} \otimes V_{2} \otimes V_{3}$ of vector spaces of the matrices $M_{i} \in V_{i}$ :

$$
\sigma_{13}\left(\begin{array}{c}
M_{1} \\
M_{2} \\
M_{3}
\end{array}\right)=\left(\begin{array}{c}
M_{3} M_{1} M_{3}^{-1} \\
M_{2} \\
M_{3}
\end{array}\right) .
$$

The clockwise exchange occuring for $\operatorname{Arg}(m)>0$ (Fig.(枯)) yields another operator:

$$
\sigma_{13}^{\prime}=\left(\begin{array}{c}
M_{1} \\
M_{2} \\
M_{3}
\end{array}\right)=\left(\begin{array}{c}
M_{1} \\
M_{2} \\
M_{1}^{-1} M_{3} M_{1}
\end{array}\right) .
$$

These two inequivalent operators are depicted in Fig.(6), in the standard notation for braids, and are actually related by $\sigma_{i j}^{\prime}=\left(\sigma_{j i}\right)^{-1}$ (Note that the indices are associated to the threads in our notation). The $\sigma_{i j}$ yield a representation of the braid group $\mathcal{B}_{3}$ in the space of monodromy matrices, and satisfy the Yang-Baxter identity,

$$
\sigma_{23} \sigma_{13} \sigma_{12}=\sigma_{12} \sigma_{13} \sigma_{23},
$$

which states the associativity of braids, as shown in Fig.(7)). The required onedimensional ordering of monodromies can be recognized from the position of the cuts as they cross the $u \rightarrow \infty$ circle.

The fusion of two singularities, occurring for $\operatorname{Arg}(m)=0$, Fig. (44a), can be similarly associated to the fusion operator, which is a mapping $f_{31}: V_{1} \otimes V_{2} \otimes V_{3} \longrightarrow$ $V_{31} \otimes V_{2}$ :

$$
f_{31}\left(\begin{array}{c}
M_{1} \\
M_{2} \\
M_{3}
\end{array}\right)=\left(\begin{array}{c}
M_{3} M_{1} \\
M_{2}
\end{array}\right),
$$

where the ordering in the product $M_{c}=M_{3} M_{1}$ is determined by the invariance of $M_{\infty}$. An analogous fusion operation has been defined in conformal field theory [9] 

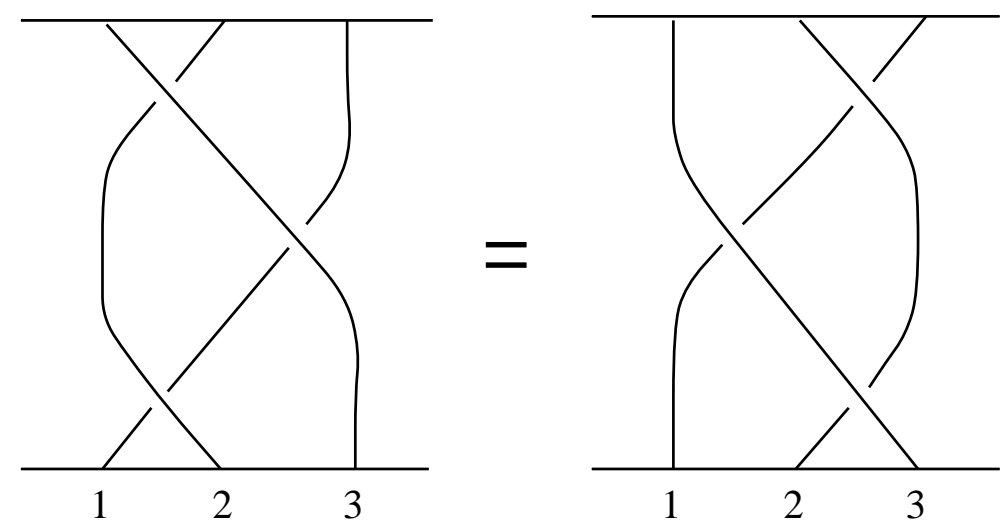

Figure 7: The Yang-Baxter identity.

and has been shown to satisfy an associativity condition with the braids, known as the Pentagonal identity. In our context, this reads:

$$
\sigma_{23} f_{12}=f_{12} \sigma_{13} \sigma_{23}
$$

and is depicted in Fig.(8). Actually, this is satisfied by our operators $\sigma_{i j}$ and $f_{i j}$. The fusion (2.18) produces a new type of singularity, whose monodromy does not belong to the BPS family (2.8): it satisfies $M_{c}^{3}=-1$ and thus corresponds to a power law singularity $u^{\alpha}$ with exponents $\alpha= \pm 1 / 6$. This scale invariant behaviour is characteristic of a superconformal theory, which occurs when two or more mutually non-local particles become simultaneously massless [11]. After fusing, the singularities split again into a quark and a monopole ones following the usual decoupling pattern, $M_{c}=M_{(0,1)} M_{(1,0)}$. In this case, there is a true discontinuity in passing from the patch $(m \sim \Lambda)$ to the asymptotic one $(m \rightarrow \infty)$. Let us remark that the fusion is a necessary consequence of the discontinuity of braiding when the mass is varied continuously from $\operatorname{Arg}(m)>0$ to $\operatorname{Arg}(m)<0$. This mathematical consistency implies the presence of the strange critical point in the moduli space along the real trajectory $0<m<\infty$; therefore, this is a peculiar physical consequence of isomonodromy.

The corresponding analysis of braiding and fusing in the $N_{f}=2,3$ theories is described in Appendix A: it confirms the properties of the braid operators discussed here, and describes the interesting patterns of quark decouplings with the associated superconformal points. 

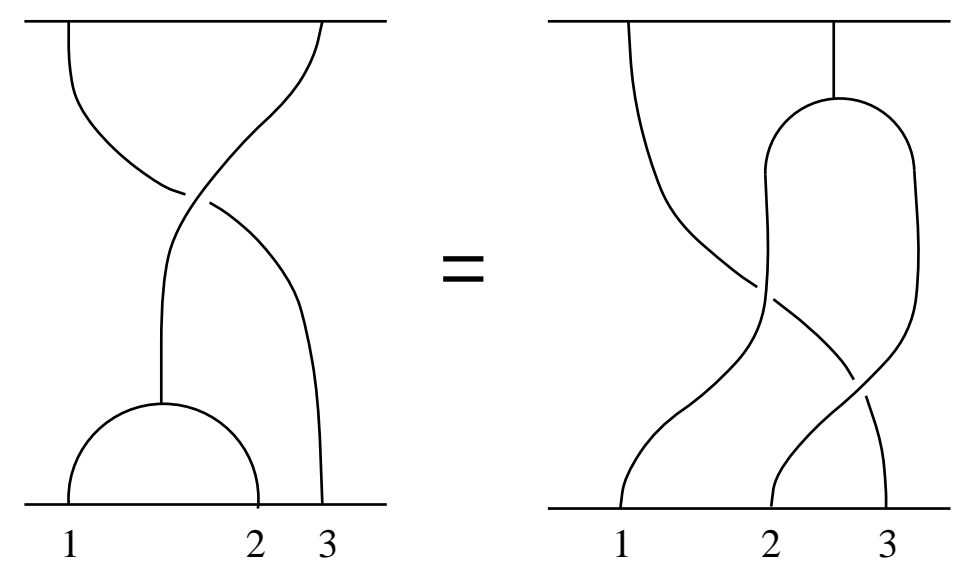

Figure 8: The Pentagonal identity.

\subsection{Complete Monodromies and Pseudo-Baryonic Quantum Numbers}

The complete monodromies of $\left(a_{D}, a\right)$ contain the additive term $\sum_{f=1}^{N_{f}}\left(q_{D f}, q_{f}\right) m_{f} / \sqrt{2}$, with $q_{D f}, q_{f} \in \mathbf{Z} / 2$, which is due to the poles occurring in the Abelian differential after their integration in $u$ [目]. These affine transformations can be represented in matrix notation as follows:

$$
\left(\begin{array}{c}
a_{D} \\
a \\
m_{f} / \sqrt{2}
\end{array}\right) \longrightarrow \mathcal{M}\left(\begin{array}{c}
a_{D} \\
a \\
m_{f} / \sqrt{2}
\end{array}\right)=\left(\begin{array}{c|c}
M & q_{D f} \\
\hline \mathbf{0} & \mathbf{1}
\end{array}\right)\left(\begin{array}{c}
a_{D} \\
a \\
m_{f} / \sqrt{2}
\end{array}\right) .
$$

The matrix $\mathcal{M}$ acts on the complete vector of quantum numbers $\mathbf{n}=\left(n_{m}, n_{e}, s_{f}\right)$ by $\mathbf{n} \longrightarrow \mathbf{n} \mathcal{M}^{-1}$, as before. As discussed by the recent literature [22] [21], the pseudobaryonic charges $\left\{s_{f}\right\}$ differ from the true baryonic charges $\left\{S_{f}\right\}$, due to the mixing of the explicit mass terms in the BPS formula with analogous pieces coming from $a_{D}\left(u, m_{f}\right)$. The baryonic charges have been computed by independent semiclassical methods in Ref. 21], while the pseudo-baryonic numbers are important missing data for the mass spectrum. In order to control these possible shifts by mass terms, the normalization of $a_{D}\left(u, m_{f}\right)$ should be better specified, such that the $\left\{s_{f}\right\}$ become unambiguous: we adopt the following decoupling condition,

$$
\lim _{m_{i} \rightarrow \infty} \mathbf{a}^{\left(N_{f}\right)}\left(u ; m_{1}, \ldots, m_{i}, \ldots\right)=\mathbf{a}^{\left(N_{f}-1\right)}\left(u ; m_{1}, \ldots, m h_{i}, \ldots\right),
$$

with $i \in(1, \ldots, f)$; this gives a recursive definition of $\mathbf{a}^{\left(N_{f}\right)}=\left(a_{D}, a\right)^{\left(N_{f}\right)}$ in terms of the well-defined $\mathbf{a}^{(0)}(u)$. Note that this limits also implies the matching of patches between the two theories at quark decoupling, as in Fig.(55d). 
The determination of the complete monodromies (2.20) requires the explicit expression of the Seiberg-Witten section [23] [24] [25], whose analytic continuation is rather difficult, in general. It follows that these monodromies, as well as the $\left\{s_{f}\right\}$ numbers, have not been completely found in the literature [26] 25: In the following, we shall determine the complete $N_{f}=1$ monodromies by using the braiding and fusing identities as equations. The corresponding analysis for $N_{f}=2,3$ is given in the Appendix A. The inputs for these equations are:

- The known $S L(2, \mathbf{Z})$ monodromies (2.11).

- The full monodromy of the quark singularity $a \sim-\epsilon m / \sqrt{2}, \epsilon= \pm 1$, which can be deduced at weak coupling from the one-loop calculation [1]:

$$
\mathcal{M}_{(0,1, \epsilon)}=\left(\begin{array}{ccc}
1 & 1 & \epsilon \\
0 & 1 & 0 \\
0 & 0 & 1
\end{array}\right), \quad \epsilon= \pm 1
$$

Note that the sign ambiguity $\epsilon$ is odd under the parity $P: a \rightarrow-a$; it is a residual gauge invariance in the moduli space which will remain undetermined: it corresponds to the choice of the square-root branch cut for $a \sim \pm \sqrt{u} \rightarrow \infty$.

- The matching of the $N_{f}$ monodromies with the $\left(N_{f}-1\right)$ ones at quark decoupling; in particular the affine terms vanish for $N_{f}=0$.

We write the full monodromies at strong coupling, Fig.(5a), as $\mathcal{M}_{(1,-1, r)}, \mathcal{M}_{(1,1, s)}$, $\mathcal{M}_{(1,0, t)}$, leaving their affine terms unknown, and plug them into the previous braid relations, Eq.(2.13) and Fig.(5d):

$$
\begin{aligned}
\mathcal{M}_{(1,0, t)} \mathcal{M}_{(1,-1, r)} \mathcal{M}_{(1,0, t)}^{-1} & =\mathcal{M}_{(0,1, \epsilon)} & \text { (quark monodromy) } \\
\mathcal{M}_{(0,1, \epsilon)}^{-1} \mathcal{M}_{(1,1, s)} \mathcal{M}_{(0,1, \epsilon)} & =\mathcal{M}_{(1,2)}^{(0)} & \left(N_{f}=0, \text { no affine term }\right) \\
\mathcal{M}_{(1,0, t)} & =\mathcal{M}_{(1,0)}^{(0)} & \left(N_{f}=0, \text { no affine term }\right)
\end{aligned}
$$

The unique solution is:

$\mathcal{M}_{(1,-1, r)}=\left(\begin{array}{ccc}0 & 1 & \epsilon \\ -1 & 2 & \epsilon \\ 0 & 0 & 1\end{array}\right), \mathcal{M}_{(1,1, s)}=\left(\begin{array}{ccc}2 & 1 & -\epsilon \\ -1 & 0 & \epsilon \\ 0 & 0 & 1\end{array}\right), \mathcal{M}_{(1,0, t)}=\left(\begin{array}{ccc}1 & 0 & 0 \\ -1 & 1 & 0 \\ 0 & 0 & 1\end{array}\right)$

$\ddagger \ddagger$ Note, however, that it is possible to identify $\left\{s_{f}\right\} \equiv\left\{S_{f}\right\}$ by choosing a boundary condition for $a_{D}^{\left(N_{f}\right)}$ different from (2.21), and then use the independent semiclassical determination of the $\left\{S_{f}\right\}$ 21. 
which yields the following monodromies at infinity and at the critical point,

$$
\mathcal{M}_{\infty}^{(1)}=\left(\begin{array}{ccc}
-1 & 3 & \epsilon \\
0 & -1 & 0 \\
0 & 0 & 1
\end{array}\right), \quad \mathcal{M}_{c}^{(1)}=\mathcal{M}_{(1,0, t)} \mathcal{M}_{(1,-1, r)}=\left(\begin{array}{ccc}
0 & 1 & \epsilon \\
-1 & 1 & 0 \\
0 & 0 & 1
\end{array}\right)
$$

Note that two further sets of identities (2.23) can be written for the other, $\mathbf{Z}_{3^{-}}$ symmetric quark decouplings, but they are automatically satisfied by this solution. The results in this Section have been compared with the literature [26] 25] 22] 221], when available; moreover, $\mathcal{M}_{c}^{(1)}$ and $\mathcal{M}_{(1,1, s)}$ have been checked by explicitly computing the Seiberg-Witten section at the critical mass in Appendix B.

Next we discuss some simple physical consequences of the result (2.24), (2.25). Following Ref. [12], we can constrain the values of the $\left\{s_{f}\right\}$ charges in the BPS spectrum by enforcing its invariance under the spectral flow at weak coupling. Namely, any weak-coupling dyon state $\mathbf{n}=(1, n, s)$, with $n \in \mathbf{Z}$ and $s$ unknown, should map into another one under the monodromy at infinity, corresponding to a rotation of the $\theta$-angle by three periods 21]: this reads $(1, n, s) \mathcal{M}_{\infty}^{-1}=-(1, n+3,-s-\epsilon)$. This flow closes on the following two sets of dyons: $\{(1,2 n, s)\}$ and $\{(1,2 n+1,-s-\epsilon)\}$, $n \in \mathbf{Z}$, with $s$ still free. This is fixed to be $s=0$ by the further condition that the dyons with even electric charge should survive in the $N_{f}=0$ theory as $m \rightarrow \infty$. In conclusion, the complete $N_{f}=1$ spectrum is:

$$
(1,2 n, 0), \quad(1,2 n+1,-\epsilon), \quad n \in \mathbf{Z}, \quad\left(N_{f}=1\right) .
$$

Thus, the pseudo-baryonic charges in the previous Eqs.(2.23) are $r=s=-\epsilon$ and $t=0$.

Further remarks concern the behaviour at the critical point. The Seiberg-Witten section is found to be $\left(a_{D}, a\right)\left(u_{c}\right)=(0,-\epsilon m / \sqrt{2})$ by requiring that the BPS mass of the two particles $(1,0,0)$ and $(1,-1,-\epsilon)$ vanishes at this point. One verifies that this value of the section is a non-trivial fixed point for the monodromy $\mathcal{M}_{c}$ in Eq.(2.25), as it should. Moreover, a non-vanishing value implies that the $W^{ \pm}$bosons $\mathbf{n}=(0, \pm 2,0)$ remain massive at this point, $m_{W}=m^{2}$, i.e. there is no restoration of the non-Abelian gauge symmetry [1].

Finally, we determine the weak-coupling spectrum of the $N_{f}=2,3$ theories. We use the corresponding monodromies at infinity computed in Appendix A, Eqs. A.4,A.9), and we get the spectra of dyon charges $\left\{\left(n_{m}, n_{e}\right)\right\}$ from Ref.[1]. We then find the spec-

\footnotetext{
$\dagger$ Note, however, that $\mathcal{M}_{c}^{3} \neq 1$, i.e. the complete monodromies no longer represent $S L(2, \mathbf{Z})$.
} 
tral flows:

$$
\begin{aligned}
& \mathcal{M}_{\infty}^{(2)}:\left(1, n, s_{1}, s_{2}\right) \quad \longrightarrow \quad\left(1, n+2,-\epsilon-s_{1}, \epsilon-s_{2}\right), \quad\left(N_{f}=2\right) ; \\
& \mathcal{M}_{\infty}^{(3)}:\left(1, n, s_{1}, s_{2}, s_{3}\right) \quad \longrightarrow \quad\left(1, n+2,-\epsilon-s_{1}, \epsilon-s_{2}, \epsilon-s_{3}\right), \quad\left(N_{f}=3\right) \text {; } \\
& \left(2,2 n+1, s_{1}, s_{2}, s_{3}\right) \quad \longrightarrow \quad\left(2,2 n+3,-2 \epsilon-s_{1}, 2 \epsilon-s_{2}, 2 \epsilon-s_{3}\right) \text {. }
\end{aligned}
$$

Let us first discuss the $N_{f}=2$ spectrum. The spectral flow maps even (odd) charge dyons within themselves; since a tower of even dyons should survive when $m_{1}$ or $m_{2}$ go to infinity, the set $\{(1,2 n, 0,0)\}$ should exist. Then, the other set $\{(1,2 n,-\epsilon, \epsilon)\}$ should also exist by consistency of the flow. Therefore, we find that the even dyons are (at least) doubly degenerate with respect to their electric and magnetic charges. Similar conditions of flow consistency and $\left(N_{f}=1\right)$ matching can be applied to the odd dyons, which also come in pairs. The $N_{f}=2$ spectrum is thus found to be:

$$
\begin{array}{ll}
(1,2 n, 0,0), & (1,2 n+1,-\epsilon, 0), \\
(1,2 n,-\epsilon, \epsilon), & (1,2 n+1,0, \epsilon),
\end{array} \quad n \in \mathbf{Z}, \quad\left(N_{f}=2\right) .
$$

Note that the symmetry under flavour permutation is achieved up to a gauge transformation $\epsilon \rightarrow-\epsilon$. Actually, within our choice of patches, the decoupling of the two quarks is associated to the monodromy decomposition $\mathcal{M}_{\infty}^{(2)}=\mathcal{M}_{(0,1, \epsilon, 0)} \mathcal{M}_{\infty}^{(0)} \mathcal{M}_{(0,1,0, \epsilon)}$. This shows that the permutation of the quarks requires a non-trivial conjugation which changes the sign of $\epsilon$.

Next, we perform a similar analysis for the $N_{f}=3$ dyon spectrum at weak coupling. There are dyons with one unit of magnetic charge which turns out to be four-fold degenerate, and, moreover, dyons with two units of magnetic charge, which can be consistently taken non-degenerate. One finds the spectrum:

$$
\begin{array}{ll}
(1,2 n, 0,0,0) & (1,2 n+1,-\epsilon, \epsilon, \epsilon) \\
(1,2 n,-\epsilon, \epsilon, 0) & (1,2 n+1,0,0, \epsilon) \\
(1,2 n,-\epsilon, 0, \epsilon) & (1,2 n, 0, \epsilon, 0) \\
(1,2 n, 0, \epsilon, \epsilon) & (1,2 n,-\epsilon, 0,0) \\
\left(2,2 n+1, r_{1}, r_{2}, r_{3}\right) &
\end{array}
$$$$
n \in \mathbf{Z}, \quad\left(N_{f}=3\right)
$$

where the pseudo-baryonic charges $r_{1}, r_{2}, r_{3} \neq 0$ of the $n_{m}=2$ dyons remain practically undetermined. Let us add a few remarks: $(i)$ The degeneracies of the $N_{f}=2,3$ spectra nicely match the dimensions of their flavour multiplets in massless limit [1] [12] 26]; ( $i i)$ The purely electric states of quarks $(0,1, \pm \epsilon, \ldots)$ and $W^{ \pm}$bosons $(0, \pm 2,0, \ldots)$ are always present in these spectra: they are fixed points of the spectral flow, as they should, and their $s_{f}$ number are not constrained, but are nevertheless known semi-classically. 
In conclusion, the complete affine monodromies have been easily found by enforcing the braiding and fusing identities. These data of the $S U(2)$ theories allow an (almost) complete determination of the weak coupling spectra and their behaviour when one or more quarks decouple. The BPS states at strong coupling are a subsets of the weak coupling spectra, with unchanged quantum numbers, which can be determined by the stability analysis of Ref.25.

\section{The Analytic Isomonodromy Problem}

In the following, we prove explicitly the isomonodromy of the Seiberg-Witten section in the simplest non-trivial case of three singularities at finite $u$, corresponding to $N_{f}=$ 1. The original approach to the isomonodromic problem [14 is based on the study of the Fuchsian second-order differential equations, which can describe holomorphic sections with two-dimensional monodromies. We shall apply this framework to the second-order equation obeyed by $\left(d a_{D} / d u, d a / d u\right)$ : its components are given by the period integrals of the first and second Abelian differentials [1], $\Pi_{1}=\oint d x / y, \Pi_{2}=$ $\oint x d x / y$, respectively, where $y(x)$ is the elliptic curve (2.5). They satisfy a system of first-order differential equations, which are called the Picard-Fuchs equations:

$$
\begin{aligned}
\frac{d \Pi_{1}}{d u} & =\frac{p_{11}(u)}{\Delta(u)} \Pi_{1}+\frac{p_{12}(u)}{\Delta(u)} \Pi_{2}, \\
\frac{d \Pi_{2}}{d u} & =\frac{p_{21}(u)}{\Delta(u)} \Pi_{1}+\frac{p_{22}(u)}{\Delta(u)} \Pi_{2},
\end{aligned}
$$

where the $p_{i j}(u)$ are polynomials in the coefficients of the elliptic curve and their derivatives [13]. Their explicit form in the $N_{f}=1$ case is, in the notation of the previous Section,

$$
\begin{aligned}
p_{11}(u) & =-p_{22}(u)=-4 u^{2}+4 u m^{2}+6 m, \\
p_{12}(u) & =6 u-8 m^{2}, \\
p_{21}(u) & =-16 m u+16 m^{3}+18, \\
\Delta(u) & =8\left(u^{3}-m^{2} u^{2}-9 m u+8 m^{3}+\frac{27}{4}\right) \\
& =8\left(u-a_{1}\right)\left(u-a_{2}\right)\left(u-a_{3}\right) .
\end{aligned}
$$

Note that we introduced the notation $a_{1}, a_{2}, a_{3}$ for the three singularities in the moduli space. It is convenient to fix two of them, say $a_{1}$ and $a_{2}$, to the points $(0,1)$ by using the $S L(2, \mathbf{C})$ transformation $u \rightarrow z=\left(u-a_{1}\right) /\left(a_{2}-a_{1}\right)$. 
The system (3.1) is equivalent to the following Fuchsian differential equation in the variable $z$ :

$$
\left[\frac{d^{2}}{d z^{2}}-q(z)\right] y(z)=0, \quad y(z)=p(z) \frac{d a}{d z}
$$

where the "potential" $q(z)$ is,

$$
\begin{aligned}
q(z)= & -\frac{1}{4 z^{2}}-\frac{1}{4(z-1)^{2}}-\frac{1}{4(z-\xi)^{2}}+\frac{3}{4(z-\eta)^{2}} \\
& +\frac{\beta_{1}}{z}+\frac{\beta_{2}}{z-1}+\frac{\beta_{3}}{z-\xi}+\frac{\beta_{4}}{z-\eta}, \\
\text { with } \quad & \xi \equiv \frac{a_{3}-a_{1}}{a_{2}-a_{1}}, \quad \eta \equiv \frac{a_{4}-a_{1}}{a_{2}-a_{1}}, \quad a_{4} \equiv \frac{4}{3} m^{2} .
\end{aligned}
$$

This equation has four regular singularities in $0,1, \xi$ and $\infty$. Moreover, one finds another one at $z=\eta$, but this does not actually correspond to a singularity of the solution $y(z)$. This is the so-called apparent singularity, a characteristic feature of isomonodromy [14]: actually, a simple counting argument shows that the Fuchsian equation with only physical singularities would not have enough parameters for allowing the monodromy matrices to be independent of the singularity positions. The effective coupling constant,

$$
\tau(u)=\frac{d a_{D}}{d a}=\frac{\theta_{\mathrm{eff}}(u)}{\pi}+i \frac{8 \pi}{g_{\mathrm{eff}}^{2}(u)},
$$

is given by the ratio of the two independent solutions of the Fuchsian equation; moreover, its Schwarzian is proportional to the potential:

$$
q(z)=-\frac{1}{2}\{\tau, z\}, \quad\{\tau, z\}=\frac{\tau^{\prime \prime \prime}}{\tau^{\prime}}-\frac{3}{2}\left(\frac{\tau^{\prime \prime}}{\tau^{\prime}}\right)^{2} .
$$

A simple consequence of these formulae is that $\tau^{\prime}(\eta)=0$, namely the apparent singularity is actually a saddle point.

The coefficients $\beta_{i}$ in (3.4) are called the accessory parameters; their expressions for the Seiberg-Witten solution can be obtained from the previous formulae:

$$
\begin{aligned}
& \beta_{1}(\xi, \eta)=-\frac{13}{36}-\frac{13}{36} \frac{1}{\xi}+\frac{5}{12} \frac{1}{\eta}+\frac{1}{12} \frac{\eta}{\xi} \\
& \beta_{2}(\xi, \eta)=\frac{13}{36}-\frac{13}{36} \frac{1}{\xi-1}+\frac{5}{12} \frac{1}{\eta-1}-\frac{1}{12} \frac{\eta-1}{\xi-1} \\
& \beta_{3}(\xi, \eta)=\frac{13}{36} \frac{1}{\xi}+\frac{13}{36} \frac{1}{\xi-1}+\frac{5}{12} \frac{1}{\eta-\xi}+\frac{1}{12} \frac{\eta-1}{\xi-1}-\frac{1}{12} \frac{\eta}{\xi} \\
& \beta_{4}(\xi, \eta)=-\frac{5}{12}\left(\frac{1}{\eta}+\frac{1}{\eta-1}+\frac{1}{\eta-\xi}\right) .
\end{aligned}
$$


Moreover, the movable and apparent singularities, respectively $\xi$ and $\eta$, are not independent in this solution, which has a single parameter $m$. Their relation can be obtained by analysing $\Delta\left(a_{4}\right)$ in (2.7) as a function of the singularities $a_{i}$, together with the derivatives $\Delta^{\prime}\left(a_{4}\right)$ and $\Delta^{\prime \prime}\left(a_{4}\right)$. By comparing these polynomials, we find the relation:

$$
\left(\eta^{2}-\xi\right)^{2}-4 \eta^{2}(\eta-1)(\eta-\xi)=0
$$

Let us now discuss the conditions for isomonodromy of the solution $y(z)$ or, equivalently, of $\tau(z)$. In the general Riemann-Hilbert problem, the accessory parameters $\beta_{i}(\xi, \eta)$ are not known, because the behaviour of $y(z)$ near the singularities only determines the second-order poles in the Fuchsian equation. Moreover, the motion of the apparent singularity $\eta$ as a function of $\xi$ is also unknown. The conditions of isomonodromy provide a sufficient, although rather involved, set of differential equations for these unknowns, which we shall briefly review [14. Afterwards, we shall check that these conditions are satisfied by the Seiberg-Witten solution.

The monodromy transformation of $\tau(z)$ does not depend on $\xi$ (or equivalently on $m$ ); thus, the quantities $d \tau / d \xi$ and $d \tau / d z$ must have the same monodromy, and their ratio must be a meromorphic function $A(z)$. This implies an auxiliary equation for $y \sim d a / d z:$

$$
\frac{d \tau}{d \xi}=A(z) \frac{d \tau}{d z} \longrightarrow \frac{d y}{d \xi}=A(z) \frac{d y}{d z}+B(z) y
$$

Next, we require the compatibility of this equation with the Fuchsian (3.4): this determines,

$$
A(z)=\frac{(\eta-\xi)}{\xi(\xi-1)} \frac{z(z-1)}{(z-\eta)}, \quad B(z)=-\frac{1}{2} \frac{d A}{d z} .
$$

Moreover, it yields four first-order differential equations for the $\beta_{i}$ in the $\eta$ and $\xi$ variables and a relation between $\beta_{4}$ and $d \eta / d \xi$. These differential equations admit three first integrals: one of these amounts to the condition for a regular singularity at infinity,

$$
\sum_{i=1}^{4} \beta_{i}=0
$$

Another integral determines the second-order pole at infinity,

$$
\beta_{2}+\beta_{3} \xi+\beta_{4} \eta=-\frac{1}{4}
$$

and the last one forbids a logarithmic behaviour of the solution near the apparent singularity $z=\eta$. This condition implies:

$$
\beta_{4}^{2}=-\frac{1}{4} \frac{1}{\eta^{2}}-\frac{1}{4(\eta-1)^{2}}-\frac{1}{4(\eta-\xi)^{2}}+\frac{\beta_{1}}{\eta}+\frac{\beta_{2}}{\eta-1}+\frac{\beta_{3}}{\eta-\xi} .
$$


These three algebraic conditions can be used to reduce the system of four differential isomonodromy conditions to a single non-linear differential equation for $\eta(\xi)$, which is the Painlevè VI equation,

$$
\begin{aligned}
\frac{d^{2} \eta}{d \xi^{2}} & =\frac{1}{2}\left(\frac{1}{\eta}+\frac{1}{\eta-1}+\frac{1}{\eta-\xi}\right)\left(\frac{d \eta}{d \xi}\right)^{2}-\left(\frac{1}{\xi}+\frac{1}{\xi-1}+\frac{1}{\eta-\xi}\right) \frac{d \eta}{d \xi} \\
& +\frac{1}{2} \frac{\eta(\eta-1)}{\xi(\xi-1)(\eta-\xi)}
\end{aligned}
$$

Note that the parameters of this equation have been set to the specific values of this problem.

We now verify that the $\left(N_{f}=1\right)$ Seiberg-Witten solution satisfies these analytic conditions of isomonodromy. Indeed, the coefficients $\beta_{i}(\xi, \eta)$ (3.7) deduced from the Picard-Fuchs equations can be shown to fulfil the first integrals (3.11,3.12, 3.13); in the third equation, one should use the polynomial relation for $\eta(\xi)$ (3.8). Furthermore, this relation is also found to be a rather remarkable complete integral of the Painlevè equation. This concludes the proof of isomonodromy.

As discussed in the Introduction, this property is rather intuitive, because the Seiberg-Witten section is specified by an elliptic curve (2.5), whose parameters can be continuously deformed. Nevertheless, it is nice to have a complete analytic proof. Moreover, the first-order differential equation in the mass parameter (3.9, 3.10) is a new result of this Section: this is an auxiliary equation for the Picard-Fuchs system (3.1) which expresses the isomonodromy property; similar equations also exist for the Seiberg-Witten solution with general gauge group and may have important consequences (see Ref.[7] in this respect).

\section{Isomonodromy and Conformal Blocks}

In Section 2, we have shown that the Seiberg-Witten holomorphic sections support braiding and fusing operations which are similar to those found by Moore and Seiberg for the conformal blocks of rational conformal field theories 9]. In this Section, we try to express directly the Seiberg-Witten sections as the blocks of a suitable conformal theory. Clearly, the conformal blocks are meant to be an operator representation for the holomorphic sections - the two-dimensional fields do not carry any immediate physical meaning.

Is there a general correspondence between isomonodromy and conformal symmetry? On one hand, all the conformal blocks yield isomonodromic holomorphic sections. 
This is a consequence of: $(i)$ the locality of the operator product expansion of the conformal fields, and ( $i i)$ the (generalized) Fuchsian differential equations satisfied by the blocks, which originate from the chiral algebra in the rational conformal field theory. On the other hand, isomonodromic sections might be non-conformal invariant, yet being analytic: an example is given by some correlators of the two-dimensional Ising model off-criticality [5]. However, there is more to say on this subject; in Appendix C, we recall some known results on the relations among isomonodromy, integrability and conformal symmetry [5] 27]. For the sake of simplicity, let us postpone this general discussion and proceed to present an explicit correspondence.

Let us try to express $\left(d a_{D} / d u, d a / d u\right)$ in the simplest cases of two singularities at finite $u$. These are the $N_{f}=0$ section and the $N_{f}=1,2,3$ ones at the mass values where $\left(N_{f}+1\right)$ singularities merge in a maximal superconformal point $\left(N_{f}, 1\right)$ [1] (these are computed in Appendix B). We compare them with the four-point conformal blocks of the Virasoro minimal models [3],

$$
\mathcal{F}_{p}(z)=\left\langle\phi_{1}(z) \phi_{2}(0) \phi_{3}(1) \phi_{4}(\infty)\right\rangle_{p}, \quad p=1,2
$$

where $z=\left(u-a_{1}\right) /\left(a_{2}-a_{1}\right)$ is the usual rescaled variable $\left(\operatorname{Re}\left(a_{2}-a_{1}\right)=\left(a_{2}-a_{1}\right)<0\right)$; the dimensions of the fields and central charge are:

$$
c\left(p, p^{\prime}\right)=1-6 \frac{\left(p-p^{\prime}\right)^{2}}{p p^{\prime}}, \quad h_{r, s}=\frac{\left(r p^{\prime}-s p\right)^{2}-\left(p-p^{\prime}\right)^{2}}{4 p p^{\prime}}
$$

The two-dimensional monodromies imply that the field $\phi_{1}(z)$ has two-dimensional operator-product expansion with the other fields: this identifies $\phi_{1}=\phi_{1,2}$, i.e. $(r, s)=$ $(1,2)$ in the Kac table (4.2); the other fields should form a closed operator-product subalgebra with $\phi_{1,2}$, and then belong to the set $\{(1, n), n=1,2,3, .$.$\} . For example,$

$$
\phi_{1,2}(z) \phi_{1, n}(0)=\frac{1}{z^{h_{1,2}+h_{1, n}}}\left(z^{h_{1, n-1}} \phi_{1, n-1}+z^{h_{1, n+1}} \phi_{1, n+1}\right)+\quad \text { regular } .
$$

This operator-product expansion should reproduce the singular behaviours of the Seiberg-Witten section, which are logarithmic at infinity,

$$
\phi_{1}(z) \phi_{4}(\infty) \sim z^{-1 / 2}(1+\log (z))+\ldots
$$

and at $z=1$,

$$
\phi_{1}(z) \phi_{3}(1) \sim 1+\log (z-1)+\ldots .
$$

At $(z=0)$, the behaviour is again logarithmic for the $N_{f}=0$ section, while it is a power law for $N_{f}=1,2,3$, due to the superconformal symmetry, with exponents $( \pm 1 / 6, \pm 1 / 4, \pm 1 / 3)$, respectively (see Appendix B for more details). 
The logarithmic behaviour is rather unusual in conformal field theory, but it is nevertheless possible for the non-unitary "logarithmic" theories $c\left(1, p^{\prime}\right)$. As explained in Ref. [15], a logarithmic operator-product expansion is obtained when the two fields appearing in the r.h.s. of (4.3) have the same dimension:

$$
h_{1, n-1}=h_{1, n+1} \quad \longrightarrow \quad c=c(1, n) .
$$

Actually, the theory $c\left(1, p^{\prime}\right)$ may contain more than one pair of degenerate dimensions, but only the pair $\left(\phi_{1, p^{\prime}-1}, \phi_{1, p^{\prime}+1}\right)$ is obtained by the $\phi_{1,2}$ operator product expansion. Furthermore, a purely logarithmic behaviour (4.5) would require $h_{1,2}+h_{1, p^{\prime}}=h_{1, p^{\prime}-1}$, which is impossible. Nonetheless, this can be cured by adding a power law prefactor to the conformal blocks. Next, we compare the explicit form of the Seiberg-Witten section with the general expression of the minimal conformal blocks given by the Dotsenko-Fateev Coulomb gas. Actually, in Section 3 of Ref. [10], an Euler-type integral representation of the four-point block $\left\langle\phi_{n, m} \phi_{1,2} \phi_{1,2} \phi_{n, m}\right\rangle$ is given, together with its Hypergeometric expressions.

Let us first consider the $N_{f}=0$ theory. From Ref.[1], we obtain,

$$
\begin{aligned}
\frac{d a_{D}}{d z} & =-i F\left(\frac{1}{2}, \frac{1}{2}, 1 ; z\right), \\
\frac{d a}{d z} & =-\frac{1}{2}(1-z)^{-\frac{1}{2}} F\left(\frac{1}{2}, \frac{1}{2}, 1 ; \frac{1}{1-z}\right),
\end{aligned}
$$

where $z=(1-u) / 2$. By matching $d a_{D} / d z$ with the parameters in the DotsenkoFateev expression, we find the unique identification with the following blocks of the $c(1,2)=-2$ minimal model:

$$
\left(\frac{d a_{D}}{d z}, \frac{d a}{d z}\right) \sim \lim _{z_{4} \rightarrow \infty} \frac{\left\langle\phi_{12}(z) \phi_{12}(0) \phi_{12}(1) \phi_{12}\left(z_{4}\right)\right\rangle_{p}}{\left(z(1-z) z_{4}\right)^{1 / 4}}, c=c(1,2)=-2 \quad\left(N_{f}=0\right) .
$$

Note that the prefactors cancel out in the representation for the effective coupling constant $\tau=d a_{D} / d a$ as a ratio of two blocks.

The Seiberg-Witten sections of the $N_{f}=1,2,3$ theories at their critical mass values, given in Appendix B, Eqs.(B.1,B.4,B.11), can be similarly represented by the blocks involving a pair of $\phi_{1,2}$ fields and another $\phi_{1, n}$ pair. One finds:

$$
\begin{aligned}
& N_{f}=1:\left(\frac{d a_{D}}{d z}, \frac{d a}{d z}\right) \sim \frac{\left\langle\phi_{13}(z) \phi_{13}(0) \phi_{12}(1) \phi_{12}(\infty)\right\rangle_{p}}{z^{1 / 2}(1-z)^{1 / 3}}, \quad c=c(1,3)=-7, \\
& N_{f}=2:\left(\frac{d a_{D}}{d z}, \frac{d a}{d z}\right) \sim \frac{\left\langle\phi_{14}(z) \phi_{14}(0) \phi_{12}(1) \phi_{12}(\infty)\right\rangle_{p}}{z^{7 / 8}(1-z)^{3 / 8}}, \quad c=c(1,4)=-\frac{25}{2}, \\
& N_{f}=3:\left(\frac{d a_{D}}{d z}, \frac{d a}{d z}\right) \sim \frac{\left\langle\phi_{16}(z) \phi_{16}(0) \phi_{12}(1) \phi_{12}(\infty)\right\rangle_{p}}{z^{7 / 4}(1-z)^{5 / 12}}, \quad c=c(1,6)=-24 .(4)
\end{aligned}
$$


Therefore, we were able to relate the analytic properties of the Seiberg-Witten theory with those of the conformal field theory. The Moore-Seiberg braiding and fusing operators acting on the conformal fields can be applied in this case for describing the isomonodromic properties, thus improving the analysis of Section 2.

On the other hand, this equivalence of isomonodromy and conformal symmetry is only valid for the case of three singularities, i.e. the four-point blocks. We have not been able to represent the general $N_{f}=1$ section as a five-point blocks of the logarithmic minimal models: actually, the fusion rules of $\phi_{12}$ fields are consistent with the merging of singularities in critical points, and identify the fields entering the fivepoint block. However, it is impossible to match all the asymptotic behaviours with the corresponding operator product expansions.

This failure can be understood as follows. In general, we know that the SeibergWitten holomorphic sections satisfy a Fuchsian equation with $\left(3+N_{f}\right)$ regular singularities: the three-singularity solution is necessarily an Hypergeometric function, which transforms covariantly under the $S L(2, \mathbf{C})$ regular conformal transformations of the $u$-plane, and can be represented by correlator of primary conformal fields. The general $\left(3+N_{f}\right)$-singularity solutions may not have such covariance: actually, their explicit expressions or integral representations [23] [25], and their differential equations show that these sections are not $S L(2, \mathbf{C})$ covariant. For example, we can consider the representation using the Hypergeometric of argument the modular invariant function $j(\tau(u))$ [24], and find that the mapping $j(u)$ violates $S L(2, \mathbf{C})$ covariance. Furthermore, the section of the $S U(3)$ gauge theory has been expressed in terms of the generalised Hypergeometric function of two variables $F_{4}$ in Ref. 228; this is also not conformal covariant because its integral representation is not of the Euler type as the conformal blocks [10].

A possible breaking of conformal symmetry while keeping analyticity could be obtained by correlators of non-primary fields, which depend analytically on dimensionful parameters. This program can be practical only if we can characterize these new fields by other transformation properties which replace the $S L(2, \mathbf{C})$ covariance. In this respect, our approach can be related to other attempts in the literature [18] to relate the Seiberg-Witten solutions to two-dimensional topological field theories [16]. Actually these theories, as well as the matrix models and (quantum) Liouville theory [17], are further known isomonodromic systems which do not necessarily have conformal symmetry.

Finally, let us discuss the general relation between isomonodromic sections and conformal blocks given by the theory of holonomic quantum fields of the Refs. [5]. 
These Authors have analysed the $k$-dimensional isomonodromic sections in the context of the Schlesinger system of equations, which generalizes the $k=2$ analysis of the Fuchsian equation in Section 3 (see Appendix $\mathrm{C}$ for more details). They have expressed the general isomonodromic solution as a correlator of $k$-component Weyl fermions $\Psi^{\alpha}(z), \bar{\Psi}_{\beta}(z), \alpha, \beta=1, \ldots, k$, and the "twist fields" $V\left(a_{i}\right), i=1, . ., n$, which belong to a $c=k$ conformal field theory. The monodromy around the singularity $z=a_{i}$ is described by the operator-product expansion $\Psi^{\alpha}(z) V\left(a_{i}\right)$, and the prescribed monodromy matrix $\left(M_{i}\right)_{\alpha}^{\beta}$ determines the following form of the twist field:

$$
V\left(a_{i}\right)=: \mathcal{P} \exp \left(-\int_{\infty}^{a_{i}} d y\left(\log M_{i}\right)_{\alpha}^{\beta} \bar{\Psi}_{\beta}(y) \Psi^{\alpha}(y)\right):
$$

For general, non diagonal $M_{i}$ matrices, this generalized vertex operator is only formally defined as a path-ordered $(\mathcal{P})$ series; therefore, its conformal covariance and locality are not ensured. We conclude that this approach is also affected by the difficulty of defining the field operators encountered before. Again, this general representation by twist fields correlators can be useful once we understand the specific covariance of these fields.

\section{Acknowledgements}

We would like to thank Kenichi Konishi for many useful discussions. This work is supported in part by the European Community Program "Training and Mobility of Researchers" FMRX-CT96-0012.

\section{Note Added}

Section 2 may have some overlap with the recent papers in the Refs. [25] 21]; we would like to note that our results were obtained independently, but the writing was delayed by other obligations.

\section{A Monodromies of the $N_{f}=2,3$ Theories}

We now generalise the results of Section 2.3 to the cases $N_{f}>1$. As for $N_{f}=1$, we vary the values of the the masses and follow the consequent motion of the singularities in the moduli space. We then use the braiding and fusing identities as equations for determining the complete monodromy matrices.

$$
\underline{N_{f}=2 \text { case }}
$$




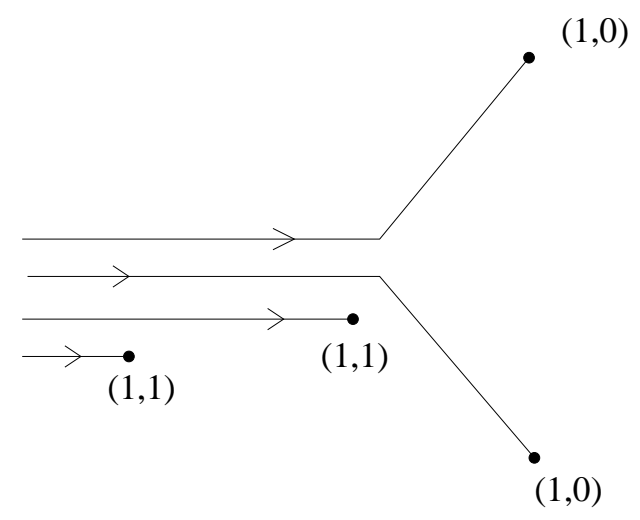

(a)

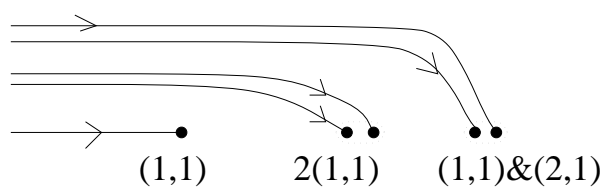

(b)

Figure 9: Singularities and cuts for the theories: (a) $N_{f}=2, m_{1}=m_{2}>0$; (b), $N_{f}=3, m_{1}=0, m_{2}=m_{3}=m>0$.

When $m_{1}=m_{2}=0$, there is a double singularity at $u=\Lambda_{2}$ which represents two massless monopoles $(1,0)$, and a double singularity at $u=-\Lambda_{2}$ of two massless dyons $(1,1)$. Their monodromies are represented, respectively, by $\mathcal{M}_{(1,0)}, \mathcal{M}_{(1,0)}^{\prime}, \mathcal{M}_{(1,1)}$, $\mathcal{M}_{(1,1)}^{\prime}$, such that $\mathcal{M}_{\infty}^{(2)}=\mathcal{M}_{(1,1)} \mathcal{M}_{(1,1)}^{\prime} \mathcal{M}_{(1,0)} \mathcal{M}_{(1,0)}^{\prime}$. If we switch on $m_{2}$ and make it growing, the two monopole singularities separate each other; one goes in the upper half plane and the other in the lower half plane (see Fig.(9 a)). The two dyon singularities separate along the real axis, one of them passes between the monopole pair and then goes to infinity: this becomes the massless quark singularity by the effect of the braiding with the lower monopole. Next, the quark cut should be rotated of $-\pi$, such that it points to $\operatorname{Re}(\mathrm{z})=+\infty$ and disappears after decoupling, leaving the $N_{f}=1$ singularities in strong coupling region: this cut rotation requires a further conjugation of the upper $(1,0)$ monopole with the quark, yielding $(1,0) \longrightarrow(1,-1)$.

The complete $N_{f}=2$ monodromies are $(4 \times 4)$ matrices, with unknown affine terms, which are plugged in the previous braid relations,

$$
\begin{array}{r}
\mathcal{M}_{(1,0)}^{-1} \mathcal{M}_{(1,1)}^{\prime} \mathcal{M}_{(1,0)}=\mathcal{M}_{(0,1,0, \epsilon)} \equiv \mathcal{M}_{\text {quark } 2}, \\
\mathcal{M}_{\text {quark } 2} \mathcal{M}_{(1,0)}^{\prime} \mathcal{M}_{\text {quark } 2}^{-1}=\mathcal{M}_{(1,-1,-\epsilon)}^{\left(N_{f}=1\right)},
\end{array}
$$

and are matched with the $N_{f}=1$ monodromies in Eq.(2.24),

$$
\mathcal{M}_{(1,1)}^{\left(N_{f}=2\right)}=\mathcal{M}_{(1,1,-\epsilon)}^{\left(N_{f}=1\right)} .
$$


We find the unique solution:

$$
\begin{array}{ll}
\mathcal{M}_{(1,1)}=\left(\begin{array}{cc|cc}
2 & 1 & -\epsilon & 0 \\
-1 & 0 & \epsilon & 0 \\
\hline \mathbf{0} & \mathbf{1}
\end{array}\right), \quad \mathcal{M}_{(1,1)}^{\prime}=\left(\begin{array}{cc|cc}
2 & 1 & 0 & \epsilon \\
-1 & 0 & 0 & -\epsilon \\
\hline \mathbf{0} & \mathbf{1}
\end{array}\right), \\
\mathcal{M}_{(1,0)}=\left(\begin{array}{cc|cc}
1 & 0 & 0 & 0 \\
-1 & 1 & 0 & 0 \\
\hline \mathbf{0} & \mathbf{1}
\end{array}\right), \quad \mathcal{M}_{(1,0)}^{\prime}=\left(\begin{array}{cc|cc}
1 & 0 & 0 & 0 \\
-1 & 1 & \epsilon & -\epsilon \\
\hline \mathbf{0} & \mathbf{1}
\end{array}\right),
\end{array}
$$

where $\epsilon$ is the same sign ambiguity of the $N_{f}=1$ case. From these it is simple to find the expressions of the monodromies at infinity and at the $(2,1)$ critical point, occurring for $m_{1}=m_{2}=\sqrt{2}$ :

$$
\begin{aligned}
\mathcal{M}_{\infty}^{(2)} & =\left(\begin{array}{cc|cc}
-1 & 2 & \epsilon & -\epsilon \\
0 & -1 & 0 & 0 \\
\hline 0 & \mathbf{0}
\end{array}\right), \\
\mathcal{M}_{c}^{(2)}=\mathcal{M}_{(1,1)}^{\prime} \mathcal{M}_{(1,0)} \mathcal{M}_{(1,0)}^{\prime} & =\left(\begin{array}{cc|cc}
0 & 1 & \epsilon & 0 \\
-1 & 0 & 0 & -\epsilon \\
\hline \mathbf{0} & \mathbf{1}
\end{array}\right) .
\end{aligned}
$$

$N_{f}=3$ case

When $m_{1}=m_{2}=m_{3}=0$, there are two singularities on the real axis of the moduli space, corresponding to four massless dyons $(1,1)$ and a $(2,1)$ massless dyon, respectively. Their monodromies are denoted by $\mathcal{M}_{(1,1)}, \mathcal{M}_{(1,1)}^{\prime}, \mathcal{M}_{(1,1)}^{\prime \prime}, \mathcal{M}_{(1,1)}^{\prime \prime \prime}$, such that $\mathcal{M}_{\infty}^{(3)}=\mathcal{M}_{(1,1)} \mathcal{M}_{(1,1)}^{\prime} \mathcal{M}_{(1,1)}^{\prime \prime} \mathcal{M}_{(1,1)}^{\prime \prime \prime} \mathcal{M}_{(2,1)}$. When $m_{3}$ acquires a real positive value, a double singularity $2(1,1)$ and the $(2,1)$ one approach each other and make a superconformal point. Afterwards, they split again with different quantum numbers, i.e. the one on the right has become the quark singularity. By allowing an imaginary part to $m_{3}$, one can resolve this motion and actually find a double braiding of $(2,1)$ around the pair $2(1,1)$, such that $(2,1) \longrightarrow(1,0)$. Therefore, the braid relation and matching conditions to impose are:

$$
\begin{array}{r}
\mathcal{M}_{(1,1)}^{\prime \prime} \mathcal{M}_{(1,1)}^{\prime \prime \prime} \mathcal{M}_{(2,1)}\left(\mathcal{M}_{(1,1)}^{\prime \prime} \mathcal{M}_{(1,1)}^{\prime \prime \prime}\right)^{-1}=\mathcal{M}_{(0,1,0,0, \epsilon)}=\mathcal{M}_{\text {quark } 3}, \\
\mathcal{M}_{\text {quark } 3} \mathcal{M}_{(1,1)}^{\prime \prime} \mathcal{M}_{(1,1)}^{\prime \prime \prime} \mathcal{M}_{\text {quark } 3}^{-1}=\mathcal{M}_{(1,0)}^{\left(N_{f}=2\right)} \mathcal{M}_{(1,0)}^{\prime\left(N_{f}=2\right)} \\
\mathcal{M}_{(1,1)} \mathcal{M}_{(1,1)}^{\prime}=\mathcal{M}_{(1,1)}^{\left(N_{f}=2\right)} \mathcal{M}_{(1,1)}^{\prime\left(N_{f}=2\right)}
\end{array}
$$

Other braid relations can be obtained from another pattern of decoupling: by letting $m_{2}=m_{3}=m$ real and positive, a $(1,1)$ singularity, followed by a $2(1,1)$ pair, move toward the $(2,1)$ one (Fig $9 \mathrm{~b})$; the $(1,1)$ and $(2,1)$ fuse into a critical point, and 
then separate, going in the upper and lower half-plane, respectively. It is simple to see that, after the separation, the singularity going downwards is a monopole $(1,0)$, while the other one is the dyon $(1,1)$. The $2(1,1)$ singularity proceeds to the right and passes between these two; the braiding rules show that this becomes a double quark singularity. By decoupling these quarks to the right, there remain the $N_{f}=1$ singularities and we find:

$$
\mathcal{M}_{\infty}^{(3)}=\mathcal{M}_{\infty}^{(1)} \mathcal{M}_{\text {quark } 2} \mathcal{M}_{\text {quark } 3}
$$

The corresponding braid relations and matching conditions are,

$$
\begin{aligned}
\mathcal{M}_{(1,1)}^{\prime \prime \prime} \mathcal{M}_{(2,1)}\left(\mathcal{M}_{(1,1)}^{\prime \prime \prime}\right)^{-1} & =\mathcal{M}_{(1,0)}^{\left(N_{f}=1\right)} \\
\mathcal{M}_{\text {quark } 2} \mathcal{M}_{\text {quark } 3} \mathcal{M}_{(1,1)}^{\prime \prime \prime}\left(\mathcal{M}_{\text {quark } 2} \mathcal{M}_{\text {quark } 3}\right)^{-1} & =\mathcal{M}_{(1,-1)}^{\left(N_{f}=1\right)} \\
\mathcal{M}_{(1,1)} & =\mathcal{M}_{(1,1)}^{\left(N_{f}=1\right)}
\end{aligned}
$$

The equation (A.5) and (A.7) are sufficient to determine all the affine terms for the monodromy matrices; the result is:

$$
\begin{aligned}
\mathcal{M}_{(1,1)}=\left(\begin{array}{cc|ccc}
2 & 1 & -\epsilon & 0 & 0 \\
-1 & 0 & \epsilon & 0 & 0 \\
\hline \mathbf{0} & & \mathbf{1}
\end{array}\right) & , \quad \mathcal{M}_{(1,1)}^{\prime}=\left(\begin{array}{cc|ccc|ccc}
2 & 1 & 0 & \epsilon & 0 \\
-1 & 0 & 0 & -\epsilon & 0 \\
\hline \mathbf{0} & \mathbf{1} &
\end{array}\right), \\
\mathcal{M}_{(1,1)}^{\prime \prime}= & , \quad \mathcal{M}_{(1,1)}^{\prime \prime \prime}=\left(\begin{array}{cc|cc|ccc}
2 & 1 & -\epsilon & \epsilon & \epsilon \\
-1 & 0 & 0 & 0 & -\epsilon \\
\hline \mathbf{0} & \mathbf{1} & 0 & \epsilon & -\epsilon & -\epsilon \\
\hline \mathbf{0} & & \mathbf{1}
\end{array}\right), \\
\mathcal{M}_{(2,1)} & =\left(\begin{array}{cc|ccc}
3 & 1 & -\epsilon & \epsilon & \epsilon \\
-4 & -1 & 2 \epsilon & -2 \epsilon & -2 \epsilon \\
\hline \mathbf{0} & & \mathbf{1} &
\end{array}\right) .
\end{aligned}
$$

From these, we calculate the monodromies at the infinity and at the critical point $(3,1)$ :

$$
\begin{aligned}
& \mathcal{M}_{\infty}^{(3)}=\left(\begin{array}{cc|ccc}
-1 & 1 & \epsilon & -\epsilon & -\epsilon \\
0 & -1 & 0 & 0 & 0 \\
\hline \mathbf{0} & & \mathbf{1} &
\end{array}\right), \\
& \mathcal{M}_{c}^{(3)}=\left(\begin{array}{cc|ccc}
0 & 1 & \epsilon & 0 & 0 \\
-1 & -1 & 0 & -\epsilon & -\epsilon \\
\hline \mathbf{0} & & \mathbf{1}
\end{array}\right),
\end{aligned}
$$

which are used in the text to derive the $s_{i}$ quantum numbers. 


\section{B Explicit Solutions at the Critical Masses}

In the recent literature, there have appeared rather explicit formulae for the SeibergWitten sections of the general massive $S U(2)$ theories with $N_{f}=1,2,3$ : one expression uses the Elliptic functions, after putting the elliptic curves in the Weierstrass normal form [23] 25]; another one is given by the Hypergemetric function with argument the modular invariant function $j(\tau(u))$ [24]. These expressions depend on the moduli space coordinate $u$ in a rather indirect way, such that the analytic continuations are rather difficult in general. Hereafter, we shall describe the simple cases in which the Seiberg-Witten section can be directly expressed in the $u$ variable as an integral of the Hypergeometric function, such that the derivation of the complete monodromies is rather straightforward.

These cases are characterized by the presence of only three singularities in the moduli space, when $\left(N_{f}+1\right)$ singularities fuse into the $\left(N_{f}, 1\right)$ superconformal points (in the notation of Ref.[11]). By extending the argument of Section 3, we can argue that the derivative $\left(d a_{D} / d u, d a / d u\right)$ satisfies a Fuchsian differential equation in the $u$ variable with three singularities, whose solution is necessarily Hypergeometric. We use the $S L(2, \mathbf{C})$-reduced variable $z=\left(u-a_{1}\right) /\left(a_{2}-a_{1}\right)$, with $\operatorname{Re}\left(a_{2}-a_{1}\right)=\left(a_{2}-a_{1}\right)<0$, and put the singularities in $z=0,1, \infty$. The logarithmic behaviours occur at $z=1, \infty$, and the power-law singularity is found at the critical point $z=0$. Its characteristic indices can be deduced by the corresponding monodromies matrices,

which represent the $S L(2, \mathbf{Z})$ group, in particular by the diagonalizable elements: $S$ $\left(S^{2}=-1\right)$ and $S T\left((S T)^{3}=1\right)$ or combinations of them. Form the analysis of Section 2 and Appendix A, we know that the critical monodromies are $(-T S), S$ and $(S T)$ for $N_{f}=1,2$ and 3, respectively. The corresponding indices $(a, b, c)$ of the Hypergeometric function $F(a, b, c ; z)$ are $(1 / 6,1 / 6,1 / 3),(1 / 4,1 / 4,1 / 2)$ and $(1 / 3,1 / 3,2 / 3)$, respectively.

To start with, let us describe the case $N_{f}=1$. We use the following bases of solutions:

$$
\begin{aligned}
y_{1}(\infty)= & k_{1}(-z)^{\left(-\frac{1}{2}\right)} F\left(\frac{1}{3}, \frac{2}{3}, 1 ; \frac{1}{z}\right) \\
y_{2}(\infty)= & k_{1}(-z)^{\left(-\frac{1}{2}\right)}\left[\log (-z) F\left(\frac{1}{3}, \frac{2}{3}, 1 ; \frac{1}{z}\right)\right. \\
& \left.+\sum_{n=0}^{+\infty} \frac{\left(\frac{1}{3}\right)_{n}\left(\frac{2}{3}\right)_{n}}{(n !)^{2}}\left(2 \psi(n+1)-\psi\left(n+\frac{1}{3}\right)-\psi\left(n+\frac{2}{3}\right)\right)\left(\frac{1}{z}\right)^{n}\right], \\
y_{1}(1)= & k_{1}(-z)^{\left(-\frac{1}{6}\right)} F\left(\frac{1}{3}, \frac{1}{3}, 1 ; 1-z\right),
\end{aligned}
$$




$$
\begin{aligned}
y_{2}(1)= & k_{1}(-z)^{\left(-\frac{1}{6}\right)}\left[\log (1-z) F\left(\frac{1}{3}, \frac{1}{3}, 1 ; 1-z\right)\right. \\
& \left.+2 \sum_{n=0}^{+\infty} \frac{\left(\frac{1}{3}\right)_{n}\left(\frac{1}{3}\right)_{n}}{(n !)^{2}}\left(\psi\left(n+\frac{1}{3}\right)-\psi(n+1)\right)(1-z)^{n}\right], \\
y_{1}(0)= & k_{1}(-z)^{\left(-\frac{1}{6}\right)} F\left(\frac{1}{3}, \frac{1}{3}, \frac{2}{3} ; z\right), \\
y_{2}(0)= & k_{1}(-z)^{\left(-\frac{1}{6}\right)} F\left(\frac{2}{3}, \frac{2}{3}, \frac{4}{3} ; z\right),
\end{aligned}
$$

which have simple monodromy transformations around each point. For example, around $z=\infty$ we have:

$$
y_{1}(\infty) \rightarrow-y_{1}(\infty) \quad y_{2}(\infty) \rightarrow-y_{2}(\infty)-2 i \pi y_{1}(\infty)
$$

The three solutions in (B.1) are related by the linear transformations:

$$
\begin{aligned}
& y_{1}(\infty)=\frac{1}{2\left(\psi\left(\frac{1}{3}\right)-\psi\left(\frac{2}{3}\right)\right)}\left(\frac{\Gamma\left(\frac{2}{3}\right)^{2}}{\Gamma\left(\frac{4}{3}\right)} y_{2}(0)-\frac{\Gamma\left(\frac{1}{3}\right)^{2}}{\Gamma\left(\frac{2}{3}\right)} y_{1}(0)\right) \\
& y_{2}(\infty)=\frac{1}{2}\left(\frac{\Gamma\left(\frac{2}{3}\right)^{2}}{\Gamma\left(\frac{4}{3}\right)} y_{2}(0)+\frac{\Gamma\left(\frac{1}{3}\right)^{2}}{\Gamma\left(\frac{2}{3}\right)} y_{1}(0)\right) \\
& y_{1}(\infty)=e^{\mp i \frac{\pi}{3}} y_{1}(1)-\frac{\sqrt{3}}{2 \pi}\left(1-e^{\mp i \frac{\pi}{3}}\right) y_{2}(1), \\
& y_{2}(\infty)=-\frac{\pi}{\sqrt{3}} e^{\mp i \frac{\pi}{3}} y_{1}(1)-\frac{1}{2}\left(1+e^{\mp i \frac{\pi}{3}}\right) y_{2}(1)
\end{aligned}
$$

In the last transformation, the upper (lower) sign refers to the case $\operatorname{Im} z>0$ (Im $z<$ $0)$.

The expressions for $d a / d z$ and $d a_{D} / d z$ are linear combinations of this basis which satisfy the asymptotics and reproduce the expected monodromies: the asymptotics $d a / d u=\epsilon / 2 \sqrt{2 u}+O\left(u^{-3 / 2}\right)$ at $z \propto-u \rightarrow-\infty$ identifies $d a / d z=y_{1}(\infty)$ with $k_{1}=$ $\epsilon \sqrt{3} m_{c} / 2 \sqrt{2}$, upon using the critical value of the mass $m_{c}=3 / 2$ and $\left(a_{1}-a_{2}\right)=27 / 4$ obtained from $\Delta(u)$ in Eq.(2.7); moreover, the sign $\epsilon= \pm 1$ accounts for the choice of the square-root for $u \sim 2 a^{2}$. The linear combination for $d a_{D} / d z$ is determined by the monodromies at infinity (2.12) and at $z=0$ : in the latter case, one applies the transformation of basis $(\mathbb{B} .3)$. The result is:

$$
\begin{aligned}
\frac{d a_{D}}{d z} & =\frac{1}{2} y_{1}(\infty)+\frac{3 i}{2 \pi} y_{2}(\infty), \quad\left(k_{1}=\epsilon \frac{\sqrt{3} m_{c}}{2 \sqrt{2}}\right) \\
\frac{d a}{d z} & =y_{1}(\infty)
\end{aligned}
$$

Next, $a$ and $a_{D}$ can be represented as contour integrals in the $u$-plane of the Hypergeometric functions; the integration constant for $a$ is determined by its asymptotic 
behaviour at infinity. The other boundary condition is chosen at the critical point: $a_{D}(0)=0$ for any $N_{f}$. This is consistent with the choice of patches in Section 2 and Appendix A, because $a_{D}(0)=0$ is left invariant by the action of all the critical monodromies. Therefore, we define,

$$
a=\int_{-\infty}^{z} d z \frac{d a}{d z}, \quad a_{D}=\int_{0}^{z} d z \frac{d a_{D}}{d z}
$$

The complete monodromy matrices for $\left(a, a_{D}\right)$ can be now computed as follows: one performs the analytic continuation of the integrand and deforms the integration contour accordingly. For the monodromy at infinity, we find,

$$
\begin{aligned}
z \sim \infty: \quad a_{D} & \rightarrow-a_{D}+3 a-2 \int_{-\infty}^{0} \frac{d a_{D}}{d z}, \\
a & \rightarrow-a .
\end{aligned}
$$

The affine terms are tabulated integrals of the Hypergeometric [29]:

$$
\begin{aligned}
\int_{-\infty}^{0} d z \frac{d a}{d z}= & k_{1} \frac{\sqrt{3}}{2 \pi}\left[\frac{\Gamma\left(\frac{1}{3}\right)^{2}}{\Gamma\left(\frac{2}{3}\right)} \int_{-\infty}^{0} d z(-z)^{\left(-\frac{1}{6}\right)} F\left(\frac{1}{3}, \frac{1}{3}, \frac{2}{3} ; z\right)-\right. \\
& \left.-\frac{\Gamma\left(\frac{2}{3}\right)^{2}}{\Gamma\left(\frac{4}{3}\right)} \int_{-\infty}^{0} d z(-z)^{\left(-\frac{1}{6}\right)} F\left(\frac{2}{3}, \frac{2}{3}, \frac{4}{3} ; z\right)\right]=-\frac{2 k_{1}}{\sqrt{3}}=-\epsilon \frac{m_{c}}{\sqrt{2}}
\end{aligned}
$$

and similarly,

$$
\int_{-\infty}^{0} d z \frac{d a_{D}}{d z}=-\frac{\epsilon}{2} \frac{m_{c}}{\sqrt{2}}
$$

Therefore, the monodromy at infinity is found to agree with the result of the algebraic approach (2.25) of Section 2.3. This is also the case for the monodromy around the critical point, which is similarly found by using $(\mathbb{B} .3)$. The monodromy around the third point $z=1$ is slightly more subtle: it depends on the base point, since the cut from $z=0$ passes by this point. Upon using the corresponding two transformations of basis in (B.3), we find the monodromies,

$$
\begin{aligned}
z \sim 1, & \operatorname{Im} z>0: \\
a_{D} \rightarrow & -a_{D}+4 a-4 \int_{-\infty}^{0} d z \frac{d a}{d z}+2 \int_{0}^{1} d z \frac{d\left(a_{D}-2 a\right)}{d z}=-a_{D}+4 a \\
a \rightarrow & -a_{D}+3 a-2 \int_{-\infty}^{0} d z \frac{d a}{d z}+\int_{0}^{1} d z \frac{d\left(a_{D}-2 a\right)}{d z}=-a_{D}+3 a \\
z \sim 1, & \operatorname{Im} z<0: \\
a_{D} \rightarrow & 2 a_{D}+a-\int_{-\infty}^{0} d z \frac{d a}{d z}-\int_{0}^{1} d z \frac{d\left(a+a_{D}\right)}{d z}=2 a_{D}+a-\epsilon \frac{m_{c}}{\sqrt{2}} \\
a & \rightarrow-a_{D}+\int_{-\infty}^{0} d z \frac{d a}{d z}+\int_{0}^{1} d z \frac{d\left(a+a_{D}\right)}{d z}=-a_{D}+\epsilon \frac{m_{c}}{\sqrt{2}} .
\end{aligned}
$$


The affine part can be evaluated thanks to:

$$
\int_{0}^{1} d z \frac{d\left(a_{D}-2 a\right)}{d z}=-2 \epsilon \frac{m_{c}}{\sqrt{2}}, \quad \int_{0}^{1} d z \frac{d\left(a_{D}+a\right)}{d z}=2 \epsilon \frac{m_{c}}{\sqrt{2}} .
$$

In conclusion, the second of these transformations checks again the result of Section 2.3 , Eq.(2.24).

By using the same methods for the $N_{f}=2$ and $N_{f}=3$ theories, we have found the explicit solutions for the mass values $m_{c}^{\prime}=m_{1}=m_{2}=\sqrt{2}$, implying the critical point $(2,1)$, and the values $m_{c}^{\prime \prime}=m_{1}=m_{2}=m_{3}=1$ for the $(3,1)$ point, respectively [11]. These read:

$$
\begin{aligned}
N_{f}= & 2: \\
\frac{d a}{d z}= & k_{2}(-z)^{\left(-\frac{1}{2}\right)} F\left(\frac{1}{4}, \frac{3}{4}, 1 ; \frac{1}{z}\right), \\
\frac{d a_{D}}{d z}= & k_{2} \frac{i}{\pi}(-z)^{\left(-\frac{1}{2}\right)}\left[\log (-z) F\left(\frac{1}{4}, \frac{3}{4}, 1 ; \frac{1}{z}\right)\right. \\
& \left.+\sum_{n=0}^{+\infty} \frac{\left(\frac{1}{4}\right)_{n}\left(\frac{3}{4}\right)_{n}}{(n !)^{2}}\left(2 \psi(n+1)-\psi\left(n+\frac{1}{4}\right)-\psi\left(n+\frac{3}{4}\right)\right)\left(\frac{1}{z}\right)^{n}\right], \\
N_{f}= & 3: \\
\frac{d a}{d z}= & k_{3}(-z)^{\left(-\frac{1}{2}\right)} F\left(\frac{1}{6}, \frac{5}{6}, 1 ; \frac{1}{z}\right), \\
\frac{d a_{D}}{d z}= & -k_{3} \frac{1}{2}(-z)^{\left(-\frac{1}{2}\right)} F\left(\frac{1}{6}, \frac{5}{6}, 1 ; \frac{1}{z}\right)+k_{3} \frac{i}{2 \pi}(-z)^{\left(-\frac{1}{2}\right)}\left[\log (-z) F\left(\frac{1}{6}, \frac{5}{6}, 1 ; \frac{1}{z}\right)\right. \\
& \left.+\sum_{n=0}^{+\infty} \frac{\left(\frac{1}{6}\right)_{n}\left(\frac{5}{6}\right)_{n}}{(n !)^{2}}\left(2 \psi(n+1)-\psi\left(n+\frac{1}{6}\right)-\psi\left(n+\frac{5}{6}\right)\right)\left(\frac{1}{z}\right)^{n}\right] \cdot \quad(\mathrm{B} .11)
\end{aligned}
$$

The proportionality constants are found to be:

$$
k_{2}=\epsilon \frac{m_{c}^{\prime}}{\sqrt{2}}, \quad k_{3}=\epsilon \frac{3 \sqrt{3}}{4} \frac{m_{c}^{\prime \prime}}{\sqrt{2}} .
$$

The section $\left(a_{D}, a\right)$ is again defined by the contour integrals in Eq.(B.5) and the monodromies are found by the same approach. The results check the algebraic methods of Appendix A, Eqs.(A.3) and (A.8), in the cases of equal masses.

\section{Isomonodromy, Integrability and Conformal Sym- metry}

In this Appendix, we recall some useful results in the literature [5] [27] which may clarify the general relations among these subjects. An isomonodromic holomorphic 
section $Y^{\alpha}\left(z ; a_{i}\right)$ with $n$ singular points $\left(a_{1}, \ldots, a_{n}\right)$ and $k$-dimensional monodromies, $\alpha=1, \ldots, k$, can be associated to a flat connection. Consider the configuration space, which is the tensor space $\mathbf{C}^{n+1}$ for $z$ and the singularity positions $\left\{a_{i}\right\}$, and represent the holomorphic section as a path-ordered exponential of a connection $\mathcal{A}$ in configuration space,

$$
Y\left(z ; a_{i}\right)=\mathcal{P} \exp \left(\oint_{\gamma} \mathcal{A}\right)
$$

where the open path $\gamma$ runs from infinity to the point $\left(z ; a_{1}, \ldots, a_{n}\right)$. This identification makes sense only if the path-ordered expression in the r.h.s. is independent of shape of the path, and thus depends on its end-point only. This implies that $\mathcal{A}$ is a flat connection, i.e. satisfies the zero-curvature condition,

$$
d \mathcal{A}+\mathcal{A} \wedge \mathcal{A}=0
$$

where,

$$
d=\frac{\partial}{\partial z} d z+\sum_{i=1}^{n} \frac{\partial}{\partial a_{i}} d a_{i}, \quad \text { and } \quad \mathcal{A}=\sum_{i=1}^{n} A_{i}\left(a_{j}\right) d \log \left(z-a_{i}\right)
$$

are the component forms of the differential and the connection, respectively. The identification (C.1) implies that $Y$ satisfies the system of first-order differential equations:

$$
(d-\mathcal{A}) Y=0
$$

The zero-curvature condition yields the integrability conditions for this system, which should be satisfied by the $(k \times k)$ matrix functions $A_{i}\left(a_{j}\right)$.

A monodromy transformation on $Y^{\alpha}\left(z ; a_{i}\right)$ is obtained by joining a closed path $\gamma_{k}^{\prime}$ to $\gamma$ in (C.1), which extends in the $z$ sub-plane of $\mathbf{C}^{n+1}$ and encircles the singularity $z=a_{k}$. The flatness of the connection implies the invariance under deformations of $\gamma_{k}^{\prime}$ off the $z$-plane in $\mathbf{C}^{n+1}$, which amount to displacements of the other singularities: this is the isomonodromy property.

This general formulation of the isomonodromic problem makes manifest a number of interesting relations:

I. The equations (C.4) and (C.2) can be analysed in components and compared with those of Section 3 based on the Fuchsian equation. The $d z$ component of (C.4) is the $k$-dimensional differential system,

$$
\frac{d}{d z} Y^{\alpha}=\sum_{i=1}^{n} \frac{\left(A_{i}\right)_{\beta}^{\alpha}}{z-a_{i}} Y^{\beta}
$$


which can be considered as a defining property for $Y$, analogue to the Picard-Fuchs equations of Section 3. The $d a_{i}$ components give auxiliary parametric equations,

$$
\frac{d}{d a_{i}} Y=-\frac{A_{i}}{z-a_{i}} Y
$$

which enforce isomonodromy. These are the analogues of the $\xi$ (mass) equation (3.9) found in the Fuchsian theory. Moreover, the zero curvature condition gives a set of non-linear compatibility conditions for the $A_{i}\left(a_{j}\right)$ known as the Schlesinger equations,

$$
\begin{aligned}
\frac{\partial}{\partial a_{i}} A_{i} & =\sum_{j=1, j \neq i}^{n} \frac{\left[A_{i}, A_{j}\right]}{a_{i}-a_{j}}, \\
\frac{\partial}{\partial a_{i}} A_{j} & =-\frac{\left[A_{i}, A_{j}\right]}{a_{i}-a_{j}}, \quad i \neq j ;
\end{aligned}
$$

these generalize the $\beta_{i}$ conditions and the Painlevè equation of Section 3.

As mentioned in Section 4, a formal solution of the system (C.4) has been given in terms of the analytic correlators of the $k$-component Weyl fermions $\Psi^{\alpha}(z), \bar{\Psi}_{\alpha}(z)$, $\alpha=1, \ldots, k$, and the twist fields $V\left(a_{i}\right)$, which belong to a $c=k$ conformal field theory [5]. It has the following form:

$$
Y_{\beta}^{\alpha}\left(z, z_{0} ; a_{i}\right)=\left(z_{0}-z\right) \frac{\left\langle\bar{\Psi}_{\beta}\left(z_{0}\right) \Psi^{\alpha}(z) V_{1}\left(a_{1}\right) \ldots V_{N}\left(a_{n}\right)\right\rangle}{\left\langle V_{1}\left(a_{1}\right) \ldots V_{N}\left(a_{n}\right)\right\rangle},
$$

where $z_{0}$ is a base point and the additional index $\beta$ specifies the choice of boundary conditions for the solutions; the form of the twist fields is given in Eq.(4.10). The derivation of this representation follows the same steps of the analysis in Section 4: one compares the operator-product expansion $\Psi(z) V\left(a_{i}\right)$ around each singularity $a_{i}$ with the given monodromy data, and then determines the field $V\left(a_{i}\right)$ by formal integration of this local expansion.

II. A general formulation of integrable systems in two dimensions can be given in terms of a flat connection on an certain space; therefore, we can consider the zero-curvature condition (C.2) arising in the isomonodromy problem as the defining equations of the associated integrable system. This is a heuristic argument for showing that isomonodromy and integrability have a common origin.

III. The correlators of Rational Conformal Field Theories often satisfy the Knizhnik-Zamolodchikov equations, which follows from the symmetry under an affine Lie algebra $\widehat{\mathcal{G}}$ (implying conformal symmetry) [3]. These equations can also be written in terms of a flat connection in configuration space, the Kohno connection [27, and 
correspond to a special case of the previous isomonodromy problem. This discussion also applies to the general Rational Conformal Field Theories without affine algebra, including the Virasoro minimal models of Section 4, because they can be obtained by the former theories via the coset construction, which replace $\mathcal{G}$ with $\mathcal{G} / \mathcal{H}$ [3].

As seen in Section 4 , the $n$-singularity section $Y\left(z, a_{i}\right)$ should be compared with a $(n+1)$-point correlator $\left\langle g_{n+1}(z) g_{1}\left(a_{1}\right) g_{n}\left(a_{n}\right)\right\rangle$, where the point $z$ is considered on the same footing as the $a_{i}$ ones, e.g. $z=a_{n+1}$. The conformal fields $g_{i}\left(a_{i}\right)$ carry a unitary representation $R_{i}$ of the Lie group $\mathcal{G}$ of dimension $d_{i}$, such that the monodromy problem is defined in the tensor space $\otimes_{i=1}^{n+1} R_{i}$ of dimension $k=\sum_{i=1}^{n+1} d_{i}$. The Lie group generators acting on $R_{i}$ are represented by $t_{i}^{a}$, satisfy $\left[t_{i}^{a}, t_{i}^{b}\right]=f^{a b c} t_{i}^{c}$, and $\left[t_{i}^{a}, t_{j}^{b}\right]=0$ for $i \neq j$. Kohno has introduced the connection $\Omega$, which is a symmetrized version of $\mathcal{A}$ :

$$
\begin{aligned}
\mathcal{A} & \equiv \mathcal{A}_{n+1}=\sum_{i=1}^{n} A_{n+1, i} d \log \left(a_{n+1}-a_{i}\right) \longrightarrow \\
\Omega & =\frac{1}{2} \sum_{j=1}^{n+1} \mathcal{A}_{j}=\frac{1}{2} \sum_{j=1}^{n+1} \sum_{i=1, i \neq j}^{n+1} A_{j i} d \log \left(a_{j}-a_{i}\right) .
\end{aligned}
$$

The component matrices are $A_{i j}=(1 / \kappa) \sum_{a=1}^{d_{A}} t_{i}^{a} \otimes t_{j}^{a}$, with $\kappa$ a parameter and $d_{A}$ the dimension of the adjoint representation. These matrices are independent on the singularity positions $a_{i}$, namely they are a special case of the Schlesinger ones in (C.3). The usual equation $(d-\Omega) Y=0 \mathrm{read}$, in components,

$$
\kappa \frac{\partial}{\partial a_{i}} Y\left(a_{1}, \ldots, a_{n+1}\right)=\sum_{j=1, j \neq i}^{n+1} \frac{t_{i}^{a} \otimes t_{j}^{a}}{a_{i}-a_{j}} Y\left(a_{1}, \ldots, a_{n+1}\right),
$$

which are indeed the Knizhnik-Zamolodchikov equations. Moreover, the corresponding zero-curvature condition (C.2) reduces to the following algebraic conditions, known as the "infinitesimal braid relations" [27,

$$
\left[A_{i j}, A_{i k}+A_{j k}\right]=0, \quad\left[A_{i j}, A_{k l}\right]=0, \quad i \neq j \neq k \neq l
$$

which are automatically satisfied due to the underlying group structure. In conclusion, the Rational Conformal Field Theories represent special isomonodromy problems with underlying affine and conformal symmetries.

\section{References}

[1] N. Seiberg and E. Witten, Nucl. Phys. B 426 (1994) 19, Nucl. Phys. B 431 (1994) 484 . 
[2] For a review, see: A. Bilal, in 61 Rencontre entre Physiciens Thóriciens et Mathématiciens, Strasbourg (1995), hep-th/9601007; M. Peskin, Duality in Supersymmetric Yang-Mills Theory, in Fields, Strings and Duality, TASI School 1996, hep-th/9702094.

[3] For a review see: P. Ginsparg, Applied Conformal Field Theory, in Fields, Strings and Critical Phenomena, Les Houches School 1988, E. Brezin and J. Zinn-Justin eds., North-Holland, Amsterdam (1990); P. Di Francesco, P. Mathieu and D. Senechal, Conformal Field Theory, Springer, Berlin (1997).

[4] N. Seiberg, Phys. Lett. B 206 (1988) 75, Phys. Lett. B 318 (1993) 469.

[5] M. Jimbo, T. Miwa, M. Sato and Y. Mori, Holonomic Quantum Fields: the Unanticipated Link between Deformation Theory of Differential Equations and Quantum Fields, in Int. Conf. Math. Phys., Lausanne, 1979, Lausanne Math. Phys. (1979) 119; M Jimbo, T. Miwa and K. Ueno, Physica 2D (1981) 306; G. Moore, Comm. Math. Phys. 133 (1990) 261.

[6] K. Takasaki and T. Nakatsu, Int. J. Mod. Phys. A 11 (1996) 5505.

[7] T. Eguchi and S.-K. Yang, Mod. Phys. Lett. A11 (1996) 131.

[8] E. Martinec and N. Warner, Nucl. Phys. B 459 (1996) 97; R. Donagi and E. Witten, Nucl. Phys. B 460 (1996) 299.

[9] G. Moore and N. Seiberg, Phys. Lett. B 212 (1988) 451, Nucl. Phys. B 313 (1989) 16, Commun. Math. Phys. 123 (1989) 77; for a review, see: G. Moore and N. Seiberg, Lectures on RCFT, proceedings of the 1989 Banff Summer school, H. C. Lee ed., Plenum Press, New York (1990).

[10] Vl. S. Dotsenko and V. A. Fateev, Nucl. Phys. B 240 (1984) 312.

[11] C. Argyres and M. Douglas Nucl. Phys. B 448 (1995) 93; P. Argyres, M. Plesser, N. Seiberg and E. Witten, Nucl. Phys. B 461 (1996) 71.

[12] A. Bilal and F. Ferrari, Nucl. Phys. B 469 (1996) 387, Nucl. Phys. B 480 (1996) 589.

[13] K. Ito and S.-K. Yang, Phys. Lett. B 366 (1996) 165.

[14] M. Yoshida, Fuchsian Differential Equations, Fried. Vieweg \& Sohn Editor, Bonn (1987); R. Fuchs, Mat. Annalen 63 (1907) 301; R. Garnier, Ann. Sci. Ecole Norm. Sup. (3) 29 (1912) 1. 
[15] V. Gurarie, Nucl. Phys. B 410 (1993) 535; M. Flohr, Int. J. Mod. Phys. A 11 (1996) 4147; J.-S. Caux, I.I. Kogan and A.M. Tsvelik, Nucl. Phys. B 466 (1996) 444.

[16] R. Dijkgraaf, H. Verlinde and E. Verlinde, Nucl. Phys. B 352 (1991) 59.

[17] P. Di Francesco, P. Ginsparg and J. Zinn-Justin, Phys. Rep. 254 (1995) 1.

[18] A. Gorsky, I. Krichever, A. Marshakov, A. Mironov and A. Morozov, Phys. Lett. B 355 (1955) 466; A. Marshakov, A. Mironov and A. Morozov, hep-th/9701123.

[19] K. Ito and S.-K. Yang, hep-th/9708017.

[20] A. Cappelli, M. Ciafaloni and P. Valtancoli, Nucl. Phys. B 369 (1992) 669.

[21] K. Konishi and H. Terao, hep-th/9707005; see also: K. Konishi, Phys. Lett. B 392 (1997) 101; M. Di Pierro and K. Konishi, Phys. Lett. B 388 (1996) 90.

[22] F. Ferrari, Phys. Rev. Lett. 78 (1997) 795.

[23] L. Alvarez-Gaume', M.Marino and F. Zamora, hep-th/9703072, hep-th/9707017.

[24] A. Brandhuber and S. Stieberger, hep-th/9609130.

[25] A. Bilal and F. Ferrari, hep-th/9706145.

[26] A. Brandhuber and S. Stieberger, Nucl. Phys. B 488 (1997) 199.

[27] T. Kohno, Ann.Inst. Fourier, 37, 4 (1987) 139.

[28] A. Klemm, W. Lerche and S. Theisen, Int. J. Mod. Phys. A 11 (1996) 1929.

[29] I. S. Gradshteyn and I. M. Ryzhik, Table of integrals, Series and Products, Academic, New York (1980). 